\title{
On the Graphs of Finite Idempotent Boolean Relation Matrices
}

\author{
David Rosenblatt
}

(July 16, 1963)

\begin{abstract}
This paper presents a graph-theoretic characterization of idempotent Boolean relation matrices of finite order. A relation-theoretic point of view is adopted in the paper. Idempotent matrices appear in the sequence of powers of any Boolean relation matrix, and are of purely theoretical as well as applied interest in connection with issues of convergence. The results provide a detailed description of the connectivity and cyclic structure of the directed graphs of idempotent matrices. The study is basically motivated by certain connectivity and flow problems which arise in the analysis of largescale information systems. The formal results are exemplified in an investigation of the asymptotic forms of a recursive model of an information system which affords a conjoint representation of processes of communication and derivation of information. A second principal application is given in a process formulation for the generation of consistent rank orderings. The relation between system design and idempotent forms is exhibited in the two applications.
\end{abstract}

\section{Introduction}

In this paper we present a graph-theoretic characterization of idempotent Boolean relation matrices of finite order, that is, matrices $S$ such that $S^{2}=S$. We adopt an approach which corresponds to that of an earlier study of the graphs and powers of finite relation matrices and stochastic matrices [9]. ${ }^{1}$ The present results essentially treat issues of detailed matrix structure.

This study is basically motivated by certain connectivity and flow problems which arise in the analysis of the structure of large-scale information systems. The formal results are exemplified in part in an investigation of the asymptotic forms of a recursive model of an information system [8, 10] (sec. 5). Additional applications are noted in the body of the paper, including among others, a Boolean process formulation of consistent rank orderings [2] (sec. 4).

\section{Definitions}

We adhere essentially to a relation-theoretic point of view in the analysis of the directed graphs of Boolean relation matrices [5]. The terminology given below corresponds in the main to that of some earlier studies $[9,10]$ and is also consistent with certain current expositions of the theory of graphs [4].

A homogeneous binary or dyadic relation defined on a finite set $\Sigma$ of elements is construed in classical fashion as any rule $\rho$ which specifies for each ordered couple $\left(a_{i}, a_{j}\right)$ of elements of $\Sigma$ that either the relation $\rho$ obtains between $a_{i}$ and $a_{j}$ (symbolically $a_{i} \rho a_{j}$ ) or that it does not obtain (symbolically $a_{i} \bar{\rho} a_{j}$ ). The one-one

\footnotetext{
'Figures in brackets indicate the literature references at the end of this paper.
}

representation of binary relations $\rho$ on $\Sigma$ by square Boolean matrices $R=\left\|r_{i j}\right\|(i, j=1, \ldots, n)$ is defined in the following way: $r_{i j}=1$ if $a_{i} \rho a_{j} ; r_{i j}=0$ if $a_{i} \bar{\rho} a_{j}$.

The graph-theoretic representation of binary relations $\rho$ on $\Sigma$ may be usefully stated in terms of the one-one representation of Boolean relation matrices by finite directed graphs. Given any square Boolean relation matrix $R$ of order $n$, the graph of $R, G(R)$, consists of $n$ objects $\alpha_{1}, \ldots, \alpha_{n}$ called vertices and the totality of ordered pairs of vertices $\vec{\alpha}_{i}, \alpha_{j}$, such that $\vec{\alpha}_{i}, \alpha_{j}$ exists in $G(R)$ if, and only if, $r_{i j}=1$ in $R$. The ordered pair or edge $\vec{\alpha}_{i}, \alpha_{j}$ is represented by an arrowed line directed from $\alpha_{i}$ to $\alpha_{j}$ with arrowhead pointing toward $\alpha_{j}$; an edge of the reflexive form $\vec{\alpha}_{i}, \alpha_{i}$ is taken to be admissible for any vertex $\alpha_{i}$ in $G(R)$ and is represented by a simple loop from $\alpha_{i}$ back to $\alpha_{i}$. A subgraph of an arbitrary graph $G$ is a subset of the edges and vertices of $G$ containing with each edge its endpoints. Given this one-one representation, it is then useful to consider the Boolean relation matrix $R(G)$ corresponding to any specified directed graph $G$. Any given subgraph $H$ of $G(H \subset G)$ may then be represented by the submatrix $R(H)$ (in the general sense of subrelation) of the relation matrix $R(G)$ corresponding to $G$.

We consider next a sequence of definitions which provide the basis for a primary classification of the directed graphs of homogeneous binary relations defined on finite sets of elements. The fundamental notion employed here is that of connectivity [4].

A vertex $\alpha$ of graph $G$ is said to be connected to a vertex $\beta$ in a subgraph $H \subset G$ if, and only if, $H$ contains edges $\overrightarrow{\alpha, \gamma_{1}}, \overrightarrow{\gamma_{1}, \gamma_{2}}, \ldots, \overrightarrow{\gamma_{m-1}, \gamma_{m}}$ and $\gamma_{m}=\beta$. It is expedient, in this context, to say that in $G, \beta$ is 
attainable or accessible from $\alpha$ by means of a directed path of length $\mathrm{m}$ steps. In a graph $G$ with subgraphs $H, K, H$ is said to be connected to $K$, if some vertex of $K$ is attainable from a vertex of $H . H$ is said to be strongly connected to $K$, if every vertex of $K$ is attainable from a single vertex of $H$.

We consider next the concept of "cyclic net." A subgraph $H \subset G$ is said to be a cyclic net of order $m$ if, and only if, $H$ contains $m(m>0)$ vertices of $G$ and each vertex of $H$ is connected (in $H$ ) to every vertex of $H$. A cyclic net $H$ of order $m$ in graph $G$ is said to be simple if, and only if, no proper subgraph $K \subset H$ is a cyclic net. A simple cyclic net will also be called a cycle. A cyclic traverse is said to exist from a vertex $\alpha$ to a vertex $\beta$ in graph $G$ if, and only if, $\beta$ is accessible from $\alpha$ by means of a directed path with at least one path-vertex contained in a cycle of $G$. An edge in $G$ is said to be cyclic if it is contained in some cycle of $G$ and is otherwise acyclic.

A cyclic net $H$ of order $m$ in graph $G$ is said to be maximal in $G$ if, and only if, every cyclic net in $G$ is a subgraph of $H$ or contains no vertex in common with $H$. A cyclic net $H$ of order $m$ in graph $G$ is said to be closed in $G$ if, and only if, $H$ is maximal in $G$ and every vertex of $G$ attainable from any vertex in $H$ is contained in $H$. A cyclic net $H$ of order $m$ in graph $G$ is said to be universal if for some positive integer $\hat{q}$ every vertex of $H$ is accessible in $\hat{q}$ steps from some (fixed) vertex $\alpha$ in $H$. A cyclic net which is universal will be called a universal net; a universal net is clearly not necessarily maximal in $G$. A cyclic net $H$ is thus universal if, and only if, there is a positive integer $q_{0}$ such that for all $q \geqslant q_{0}$, each vertex of $H$ is attainable from every vertex of $H$ in $q$ steps.

Two properties of universal nets which are of interest in the sequel are the following [9]. A cyclic net is universal if, and only if, the greatest common divisor of the orders of all cycles contained therein is unity. A cyclic net is then at once simple and universal if, and only if, it is of unit order.

\section{Idempotence}

For Boolean relation matrices of finite order, it is clear that idempotence is intrinsically connected with the issue of convergence of matrix powers (cf. [1, 3, 7 , 9]). Thus, if $R^{*}$ is in some sense the unique limit matrix in the sequence of powers of a relation matrix $R, R^{*}$ is idempotent.

For the purpose of this paper, we employ the following notation: $R, S$ square Boolean relation matrices of order $n$; union, $R \cup S$; intersection, $R \cap S$; inclusion, $R \leqslant S$; identity, $R=S$, for $R \leqslant S$ and $S \leqslant R$; proper inclusion, $R<S$, for $R \leqslant S$ and $R \neq S$; relative product or matrix product, $R S$; converse (or transpose), $\mathscr{R}$; negation (or complement), $\bar{R}$. This is also the scheme of notation employed in connection with binary relations and rectangular Boolean relation matrices.

In the following, a Boolean relation matrix $R$ will be said to be convergent in its powers if, and only if, there exists in the sequence $\left\{R^{k} ; k=1,2, \ldots\right\}$ a power $R^{m}$ such that $R^{m}=R^{m+1}$. A relation matrix $R$ will be said to be oscillatory or periodic in its powers if, and only if, there exists in the sequence $\left\{R^{k} ; k=1,2, \ldots\right\}$ a power $R^{m}$ such that $R^{m}=R^{m+p}$ where $p$ is the smallest integer for which this holds and $p>1$. Any matrix $R^{\prime}$ which appears infinitely often in the sequence of powers of a Boolean relation matrix $R$ will be called a limit matrix of $R$.

For Boolean relation matrices of finite order, the following two results are immediate [9]. First, a relation matrix is finitely either convergent or oscillatory in its powers. Second, a convergent relation matrix $R$ of order $n(n \geqslant 2)$ converges to one of the following: (i) The null matrix $\Lambda_{n}=\left\|r_{i j}\right\|, r_{i j}=0$ for all $i, j$; (ii) the universal matrix $V_{n}=\left\|r_{i j}\right\|, r_{i j}=1$ for all $i, j$; (iii) some idempotent matrix $R^{*}$ such that $\Lambda_{n}<R^{*}<V_{n}$.

It may be shown that there always exist for any Boolean relation matrix $R$ of order $n$ (periodic or convergent) three idempotent relation matrices which are functions of the powers of $R$. Let $[R]$ denote the set of limit matrices in the sequence of powers of $R$ and let $p \leqslant n$ denote the cardinal number or period of $[R]$. The elements $R_{\alpha}^{*}(\alpha=1, \ldots, p)$ of $[R]$ constitute a group under the operation of Boolean matrix multiplication with some distinguished one of these (say) $R_{1}^{*}$ the group identity [9]. The identity element $R_{1}^{*}$ is clearly idempotent. Let $R^{* *}$ denote the union of the group elements, $R^{* *}=\bigcup_{\alpha=1} R_{\alpha}^{*} . \quad R^{* *}$ is idempotent. If $R$ is convergent, then $[R]$ is evidently a unit set. Finally, the transitive closure (or "ancestral") of $R$,

$$
R_{*}=\bigcup_{h=0}^{\infty} R^{h}=\bigcup_{h=0}^{n-1} R^{h}
$$

is idempotent; here $R^{0} \equiv I_{n}$, the identity relation matrix of order $n$. The three idempotent relation matrices satisfy the relation $R_{1}^{*} \leqslant R^{* *} \leqslant R_{*}$ and are in general distinct (see Corollary 2a). If $R$ is convergent, there are at most two distinct matrices. If $I_{n} \leqslant R$ so that $R$ is convergent, the three matrices coincide identically. The preceding idempotent forms are of interest in connection with the recursive model of an information system (sec. 5).

The conditions for the convergence of a Boolean relation matrix of order $n(n \geqslant 2)$ are stated below in a lemma which is given without proof; this result constitutes a reformulation of Theorem 2 of [9].

Lemma 1: A Boolean relation matrix $\mathrm{R}$ of order $\mathrm{n}(\mathrm{n} \geqslant 2)$ converges in its powers if, and only if, either the graph $\mathrm{G}(\mathrm{R})$ contains no cycles or every maximal cyclic net in $\mathrm{G}(\mathrm{R})$ is universal.

In a Boolean relation matrix $R=\left\|r_{i j}\right\|(i, j=1, \ldots$, $n$ ) a matrix element $r_{i j}$ will be said to be 1 -convergent ( 0 -convergent) in the powers of $R$ if $r_{i j(\alpha)}^{*}=1(0)$ in all limit matrices $R_{\alpha}^{*}(\alpha=1, \ldots, p)$ of $R$. An element $r_{i j}$ is said to be oscillatory in the powers of $R$ if, and only if, it is neither 1-convergent nor 0-convergent. In the general case, a matrix element may be 1-convergent or 0 -convergent even though $R$ is periodic in its powers. For convenience, an element $r_{i j}$ which is not 0 -convergent will be called recurrent in the powers of $R$. The following lemmas provide a detailed account of 
these properties; since these results are essentially obvious we omit proofs.

LEMma 2a: An element $\mathrm{r}_{i j}$ of $\mathrm{R}(\mathrm{i}, \mathrm{j}=1, \ldots, \mathrm{n})$ is 0 -convergent in the powers of $\mathrm{R}$ if, and only if, there exists no cyclic traverse from vertex $\alpha_{i}$ to vertex $\alpha_{j}$ in $\mathrm{G}(\mathrm{R})$.

Lemma 2b: An element $\mathrm{r}_{i j}$ of $\mathrm{R}(\mathrm{i}, \mathrm{j}=1, \ldots, \mathrm{n})$ is recurrent in the powers of $\mathrm{R}$ if, and only if, there exist one or more cyclic traverses from vertex $\alpha_{i}$ to vertex $\alpha_{j}$ in $\mathrm{G}(\mathrm{R})$.

LEMMA 2c: $A$ recurrent element $\mathrm{r}_{i j}$ of $\mathrm{R}$ is 1-convergent in the powers of $\mathbf{R}$ if, and only if, for some positive integer $\mathrm{q}_{0}$ the cyclic traverses from vertex $\alpha_{i}$ to vertex $\alpha_{j}$ in $\mathrm{G}(\mathrm{R})$ exhibit paths of all integral lengths $\mathrm{q} \geqslant \mathrm{q}_{0}$.

It may be remarked that an element $r_{i j}$ of $R$ is 1-convergent in the powers of $R$ only if the cyclic traverses from vertex $\alpha_{i}$ to vertex $\alpha_{j}$ exhibit paths of all integer lengths $q \leqslant k(\bmod k)$ where $k$ is some cycle order of $G(R)$.

THEOREM 1: Let $\mathrm{R}$ be a convergent Boolean relation matrix of order $\mathrm{n}$. An element $\mathrm{r}_{i j}$ of $\mathrm{R}$ is l-convergent (0-convergent) if, and only if, there exists a (no) cyclic traverse from vertex $\alpha_{i}$ to vertex $\alpha_{j}$ in the graph $\mathrm{G}(\mathrm{R})$.

Proof: The result follows directly from Lemmas 1, $2 \mathrm{a}$, and $2 \mathrm{c}$, since 1 -convergence coincides with recurrence in this case.

Corollary: Let $\mathrm{R}$ be a convergent Boolean relation matrix of order $\mathrm{n}$ with limit matrix $\mathrm{R}^{*} \neq \Lambda_{n} . \mathrm{R}^{*}$ is an equivalence relation matrix if, and only if, all vertices and edges of $\mathrm{G}(\mathrm{R})$ are contained in cycles.

PROOF: $R^{*}$ is an equivalence relation matrix if, and only if, it is at once reflexive, symmetric, and transitive. The result then follows directly from the Theorem, since symmetry of $R^{*}$ requires the mutual inaccessibility of vertices in distinct maximal cyclic nets of $G(R)$.

We consider next a graph-theoretic characterization of the structure of idempotent relation matrices. For this purpose we complete the primary classification of vertices of directed graphs begun earlier. A vertex $\alpha$ in a graph $G$ will be said to be circuit free in $G$ if, and only if, $\alpha$ is contained in no cycle of $G$. We proceed to a classification of circuit free vertices.

A subgraph $J \subset G$ is a null net of order $m$ in graph $G$ if, and only if, $J$ contains $m(m>0)$ vertices circuit free in $G$ and all vertices of $J$ are connected to the same vertices of $G$. Obviously, no vertex in a null net $J$ is connected to any vertex of $J$. A null net $J$ of order $m$ in graph $G$ is said to be maximal in $G$ if, and only if, every null net in $G$ is a subgraph of $J$ or contains no vertex in common with $J$. A maximal cyclic net or a maximal null net of a graph $G$ will be called a maximal net of $G$. Every vertex of a graph $G$ is, therefore, uniquely assigned to some maximal net of $G$.

A maximal null net $N \subset G$ is said to be an articulated net in $G$ if, and only if, the following condition obtains: for every vertex $\alpha$ accessible from $N$ in $G$ there exists a cyclic traverse from $N$ to $\alpha$ in $G$. In the graph of a strong ordering relation matrix, for example, the terminal vertex constitutes an articulated net and all other vertices are in null nets of unit order which are not articulated. The concept of maximal null net is evi- dently important in the formal representation of "redundancy" in graphs or networks.

A subgraph $H \subset G$ is said to be complete in $G$ (or a complete subgraph of $G$ ) if, and only if, any vertex of $G$ which is attainable from any vertex of $H$ is attainable in one step. The null graph $G\left(\Lambda_{n}\right)$ is a complete articulated net; the universal graph $G\left(V_{n}\right)$ is the unique complete universal net of order $n$.

A graph $G$ will be called a permanent graph if, and only if, any maximal net of $G$ is either a complete articulated net or a complete universal net. A permanent graph $G$ is then, evidently, complete.

THEOREM 2: A Boolean relation matrix $\mathrm{R}$ is idempotent if, and only if, $\mathrm{G}(\mathrm{R})$ is a permanent graph.

Proof: Sufficiency. Assume $G(R)$ is a permanent graph. We show that $R$ is transitive $\left(R^{2} \leqslant R\right)$ and compact $\left(R \leqslant R^{2}\right) . \quad G(R)$ is complete so that $R^{2} \leqslant R$ follows directly. Thus, any pair of vertices $\alpha_{i}, \alpha_{j}$ of $G(R)$ are either connected in one step or not connected at all. To establish $R \leqslant R^{2}$, we show that if any $\alpha_{i}$ is connected to an $\alpha_{j}$, then it is connected to it in two and indeed any integral number of steps. Assume that $\alpha_{i}$ is connected to $\alpha_{j}$. Then either there exists a cyclic traverse from $\alpha_{i}$ to $\alpha_{j}$ in $G(R)$ in which one or both vertices are in universal nets and, consequently (since $G(R)$ is complete), in cycles of unit order, or else both vertices are in articulated nets with an intervening vertex of a universal net between them. In both cases, two-step connection (in fact $k$-step connection, $k \geqslant 2$ ) obtains. $\quad R^{2} \leqslant R$ and $R$ is idempotent.

Necessity. Assume $R$ is idempotent. Since $R^{2}$ $\leqslant R, G(R)$ is complete. By Lemma 1, any maximal cyclic net of $G(R)$ is a complete universal net. Consider any maximal null net $N$ which is connected to any vertex $\alpha$ of a maximal (null or cyclic) net $L$ in $G(R)$. By Theorem 1, there exists a cyclic traverse from $N$ to $\alpha$ of $L$ so that $N$ is a complete articulated net. $G(R)$ is, therefore, a permanent graph.

Theorem 2, in effect, states that in the graph $G(R)$ of an idempotent relation matrix $R$ a vertex $\alpha$ is connected to a vertex $\beta$ if, and only if, $\alpha$ is connected to $\beta$ in one step and there exists a cyclic traverse from $\alpha$ to $\beta$ which passes through a universal net.

COROLlaRY 2a: For any Boolean relation matrix $\mathrm{R}$ of order $\mathrm{n}$, the group union $\mathrm{R}^{* *}$ and the transitive closure $\mathrm{R}_{*}$ coincide if, and only if, every vertex of $\mathrm{G}(\mathrm{R})$ is in a cycle.

Proof: The idempotent matrices $R^{* *}$ and $R_{*}$ both have permanent graphs. $G\left(R_{*}\right)$ contains all vertex accessibilities of $G(R)$ (and also slings or auto-accessibilities for all vertices), while $G\left(R^{* *}\right)$ exhibits all recurrent vertex accessibilities of $G(R)$. Thus, $R_{*}$ $=R^{* *}$ if, and only if, all vertex accessibilities of $G(R)$ are recurrent and, in fact coincide with 1-convergences of elements of $R_{*}$ and $R^{* *}$. But this is so if, and only if, every vertex of $G(R)$ is in a cycle.

COROLlaRY 2b: For any Boolean relation matrix $\mathrm{R}$ of order $\mathrm{n}$, there exists a unique permanent graph in the sequence of graphs $\left\{\mathrm{G}\left(\mathrm{R}^{k}\right) ; \mathrm{k}=1,2, \ldots.\right\}$.

Proof: The sequence, in fact, contains at most $(n-1)^{2}+1$ distinct graphs (cf. [9]). The result follows from the Theorem and the unique idempotence of the 
identity element in any group and thus in the group of limit matrices of $R$.

To any subgraph $H$ of $G$ there corresponds a unique complementary graph $\bar{H}$ (called the complement of $H$ ) consisting of all edges in $G$ which are not in $H$. The unique subgraph of $G$ containing all the vertices of $H$ and no other, and all edges of $\bar{H}$ with both endpoints in $H$ will be called the proper complementary graph $\bar{H}^{\prime}$ (or the proper complement of $H$ ). For any Boolean relation matrix $R$, the proper complement of $G(R)$ is identified with the graph $G(\bar{R})$ of the negation $\bar{R}$ of $R$.

A set of maximal nets in a graph $G$ is said to be a chain of maximal nets if, and only if, any two distinct maximal nets in the set are strongly connected. A chain of maximal nets $H_{j}(j=1, \ldots, d)$ may then be written in the form $H_{1} \succ H_{2} \succ \ldots \succ H_{d}$, where the relation $>$ is asymmetric, transitive, and connected in the usual relation-theoretic sense.

We have the following result on complementary permanent graphs.

THEOREM 3: A graph $\mathrm{G}(\mathrm{R})$ and its complement are both permanent graphs if, and only if, $\mathrm{G}(\mathrm{R})$ consists of a chain of complete maximal nets which are alternately universal and articulated.

Proof: Sufficiency. We show that the condition of the Theorem holds for $G(\bar{R})$ if it holds for $G(R)$. Let $M$ be a maximal null (cyclic) net of $G(R)$. Then the proper complement $\bar{M}^{\prime}$ of $M$ is a maximal cyclic (null) net in $G(\bar{R})$. For if not, by augmenting $\bar{M}^{\prime}$ to form a maximal net $\bar{M}^{\prime \prime}$, the proper complement of $\bar{M}^{\prime \prime}$ would be a null (cyclic) net in $G(R)$ properly containing $M$, which is a contradiction. Moreover, if a maximal net $N$ is strongly connected to a maximal net $M$ in $G(R)$ where $N \neq M$, then $\bar{M}^{\prime}$ is strongly connected to $\bar{N}^{\prime}$ in $G(\bar{R})$. The maximal nets of $G(\bar{R})$ are thus complete and $G(\bar{R})$ also satisfies the condition of the Theorem. $G(R)$ and $G(\bar{R})$ are, therefore, permanent graphs.

Necessity. Assume $G(R)$ and its complement to be permanent graphs. We show that any two distinct maximal nets in $G(R)(G(\bar{R}))$ which are connected without an intervening maximal net between them, cannot be both universal nets or both articulated nets. There clearly must exist an intervening (maximal) universal net between any two distinct and connected articulated nets in $G(R)(G(\bar{R}))$. By virtue of the evident correspondence between maximal nets of $G(R)$ and $G(\bar{R})$, it then follows that an intervening articulated net must exist between any two distinct and connected (maximal) universal nets in $G(R)(G(\bar{R}))$. Finally, we show that any two distinct maximal nets in $G(R)(G(R))$ are strongly connected. Assume $M$ and $N$ to be maximal nets, $M \neq N$, neither of which is connected to the other in $G(R)(G(\bar{R}))$. Then the proper complements $\bar{M}^{\prime}, \bar{N}^{\prime}$ are symmetrically connected universal nets in $G(\bar{R})(G(R))$, which is a contradiction. Moreover, if $M$ is connected to $N$ in $G(R)(G(\bar{R})), M$ must be strongly connected to $N$; for if not, $G(R)$ or $G(\bar{R})$ would not be complete, which is again a contradiction. Consequently, $G(R)$ and $G(\bar{R})$ are single chains of complete maximal nets which are alternately universal and articulated.

COROLlaRY: If $\mathrm{G}(\mathrm{R})$ and its complement are both permanent graphs, $\mathrm{G}(\mathrm{R})$ is symmetric if, and only if, it is the null graph or the universal graph.

The following example provides an illustration of Theorem 3. Let $\rho$ denote the relation "less than, or equal to and odd" defined over the set of positive integers $S_{n}=\{k ; 1 \leqslant k \leqslant n\}$; thus for $x, y \in S_{n}, x \rho y$ if, and only if, $x<y$, or $x=y$ and is odd. The negation $\bar{\rho}$ of $\rho$ then denotes the relation "greater than, or equal to and even." The relations $\rho, \bar{\rho}$ are at once transitive and compact $\left(\rho \leqslant \rho^{2}, \bar{\rho} \leqslant \bar{\rho}^{2}\right)$ so that the corresponding Boolean relation matrices $R, \bar{R}$ are both indempotent. $G(R)$ and $G(\bar{R})$ consequently consist of single chains of maximal nets of unit order which are alternately universal and articulated.

Not all idempotent relation matrices are accessible as limit matrices of nontrivial convergent sequences. An idempotent Boolean relation matrix $S$ is said to have a proper power primitive if, and only if, there exists a convergent relation matrix $R$ with limit matrix $R^{*}=S$ $\neq R$ (cf. [6]).

THEOREM 4: An idempotent Boolean relation matrix $\mathrm{S}$ of order $\mathrm{n}(\mathrm{n} \geqslant 2)$ has no proper power primitive if, and only if, $\mathrm{G}(\mathrm{S})$ contains at least $\mathrm{n}-1$ maximal universal nets, and any path between distinct vertices in $\mathrm{G}(\mathrm{S})$ contains exactly one acyclic edge.

Proof: Sufficiency. Assume the condition of the Theorem holds. Consider any convergent relation matrix $R$ with limit matrix $R^{*}=S$. We show $R=S$. The maximal nets of $G(R)$ and $G(S)$ coincide identically by Theorems 1 and 2. Any acyclic edge in $G(R)$ is in $G(S)$ for any such edge involves at least one vertex in a universal net which is by Theorem 1 in $G(S)$. Conversely, any acyclic edge in $G(S)$ is in $G(R)$. For if an edge $\overrightarrow{\alpha, \beta}$ of $G(S)$ is not in $G(R)$, there must exist an intervening path connecting $\alpha$ to $\beta$ in $G(R)$. Such a path contains at most one vertex $\gamma$ for all such vertices must be in null nets if only acyclic paths of unit length exist in $G(S)$. If $\alpha, \beta$ are both in univeral nets, then $\overrightarrow{\alpha, \gamma}$ and $\overrightarrow{\gamma, \beta}$ are in $G(S)$, which violates the hypothesis. If either $\alpha$ or $\beta$ is in a null net, with $\gamma$ there exist two articulated nets, which again violates the hypothesis. Consequently $\gamma$ cannot exist and the edge $\overrightarrow{\alpha, \beta}$ is in $G(R) . \quad G(R)$ and $G(S)$ coincide and $R=S$.

Necessity. Assume $S$ is without proper power primitive. Consider the alternatives: (i) there exists a maximal net in $G(S)$ of order $m \geqslant 2$; (ii) $G(S)$ contains two or more articulated nets; (iii) there exists at least one path in $G(S)$ between distinct vertices involving two or more acyclic edges. If any of the three alternatives were to hold, it would be possible to construct a proper power primitive by adjoining (case (ii) and case (i) for null nets) or by removing (case (iii) and (i) for universal nets) a single edge in $G(S)$. The three alternatives are jointly excluded and lead to the condition of the Theorem. This completes the proof.

Theorem 4, in effect, states that an idempotent Boolean relation matrix $S$ of order $n(n \geqslant 2)$ has no proper power primitive if, and only if, $S$ contains an identity matrix of order $n-1$ and the intersection $S \cap \bar{I}_{n}$ is nilpotent, in fact $\left(S \cap \bar{I}_{n}\right)^{2}=\Lambda_{n}$. 
COROLlaRY: Every Boolean relation matrix $\mathrm{R}$ of order $\mathrm{n} \geqslant 3$ with $\mathrm{G}(\mathrm{R})$ and $\mathrm{G}(\overline{\mathrm{R}})$ both permanent graphs has a proper power primitive.

Proof: Directly by Theorems 3 and 4 (for $n=2$, the proposition fails).

We now define the graph of the sequence of powers of an arbitrary relation matrix and consider it briefly. The power graph $\Phi(R)$ of a Boolean relation matrix $R$ of order $n(n \geqslant 1)$ is given by the finite directed graph with labeled vertices corresponding to the distinct powers of $R$ and with edges existing only between successive powers. The order of $\Phi(R)$ is taken to be the cardinal number of vertices in $\Phi(R) . \quad \Phi(R)$ clearly constitutes a many-one connected graph which is predominantly one-one with the exception of a distinguished pair of vertices which are both connected in one step to a unique vertex of $\Phi(R)$. The following properties of $\Phi(R)$ are readily verified: (i) $\Phi(R)$ is of order $m \leqslant(n-1)^{2}+1 ; m=(n-1)^{2}+1$ if, and only if, $G(R)$ is a universal net of order $n(n \geqslant 2)$ containing only two cycles, one of order $n$ and the other of order $n-1$ [9]. (ii) $\Phi(R)$ contains a single cycle which is terminal and of an order given by the period of $R$ (for $R$ convergent, the period is unity). (iii) Let $S$ denote the relation matrix corresponding to $\Phi(R)$; then the idempotent power of $S$ has no proper power primitive if, and only if, the order of $\Phi(R)$ exceeds the period of $R$ by at most unity (cf. Theorems 2 and 4 ). (iv) Consider the sequence of power graphs defined recursively by $\Phi^{k}(R)=\Phi\left(\Phi^{k-1}(R)\right)$ for $k \geqslant 2$; then the order of $\Phi^{w}(R)$, where $w=(n-1)^{2}+1$, coincides with the period of $R$ (cf. (ii) above).

In the succeeding sections of the paper we treat two applications of the present results on idempotence.

\section{Application in a Process Formulation of Consistent Orderings}

As a simple application of some of the previous results, we briefly consider a stationary Boolean process formulation of rank orderings. In this approach, the particular properties of consistent order are taken to be absent (or not fully present) at the outset of the process, and are sequentially generated so as to be exhibited in complete form only at equilibrium term of the process. An ordering process or computation of this type might, for example, represent some aspects of the "process of weighing or balancing alternatives" in a problem of preference rankings (for a formally related approach to orderings, cf. [2]).

For present purposes, we select two types of consistent ordering, weak ordering and quasi-ordering. In a weak ordering $\rho$, the binary relation $\rho$ is required to be at once reflexive, transitive, and connected. Let $R$ denote the weak ordering relation matrix of order $n$ corresponding to $\rho . R$ then satisfies the following conditions: (i) $I_{n} \leqslant R$, (ii) $R^{2} \leqslant R$, (iii) $\bar{I}_{n} \leqslant R \cup \breve{R}$. $R$ is clearly idempotent and the following identity obtains: (iv) $R \cup \breve{R}=V_{n}$. The identity states the complete "order-comparability" of all elements of the domain and range of $\rho$. In a quasi-ordering, comparability is not imposed as the binary relation satisfies only the two requirements of reflexivity and transitivity; the corresponding quasi-ordering matrix $R$ is again idempotent. We exclude the case of a strong ordering $\rho$ ( $\rho$ at once irreflexive, connected, and transitive) in the present formulation for the obvious reason that any strong ordering matrix $R$ is nilpotent.

A finite sequence of successive powers of a Boolean relation matrix $R$ of order $n(n \geqslant 2)$ will be said to be a weak ordering (quasi-ordering) process with generator or primitive $R$ if, and only if, all powers are distinct, except for the ultimate and penultimate powers which coincide and are weak ordering (quasi-ordering) relation matrices. A weak ordering process or a quasiordering process is called nontrivial if the sequence contains at least three powers, and is otherwise termed trivial.

It is clear that examples of nontrivial weak ordering and quasi-ordering processes may be readily constructed. For any relation matrix $R$ such that $I_{n} \cup R$ $\neq R_{*}$, the sequence $\left\{\left(I_{n} \cup R\right)^{k} ; 1 \leqslant k \leqslant n\right\}$ contains a nontrivial quasi-ordering process in which the transitive closure $R_{*}$ is the terminal power. If in $G(R)$ of the preceding example, one at least of any two distinct vertices is accessible from the other (cf. (iv) above), then the sequence contains a nontrivial weak ordering process.

We have the following result on ordering processes.

Proposition: For any weak ordering relation matrix $\mathrm{R}$ of order $\mathrm{n} \geqslant 3$, there exists a nontrivial weak ordering process with $\mathrm{R}$ as terminal power.

PROOF: The result follows from Theorem 4 by showing that $R$ must have a proper power primitive (for $n=2$, the proposition fails). Assume the weak ordering matrix $R$ has no proper power primitive. Then $G(R)$ contains only cycles of unit order. Consider three distinct vertices $\alpha, \beta, \gamma$ and suppose $G(R)$ to contain an edge $\overrightarrow{\alpha, \beta}$. Then one of the following triads must exist in $G(R)$ : (i) $\overrightarrow{\alpha, \beta}, \overrightarrow{\beta, \gamma}, \overrightarrow{\alpha, \gamma}$; (ii) $\overrightarrow{\alpha, \beta}, \overrightarrow{\gamma, \beta}$, $\overrightarrow{\gamma, \alpha}$; (iii) $\overrightarrow{\alpha, \beta}, \overrightarrow{\gamma, \beta}, \overrightarrow{\alpha, \gamma}$. The graph then exhibits a path involving two acyclic edges, which is a contradiction. $R$ therefore has a proper power primitive and is the limit matrix of some nontrivial weak ordering process.

The earlier results (and the observations on power graphs) consequently provide a basis for the design of processes of varying length for the evolution of consistent orderings where the latter, in effect, arise in the attainment of "maximal connectivities." Although we do not consider these further here, ordering processes of a more general character may be introduced as "subrelations" of terms in sequences which converge to limit forms of non-ordering type; as may, for example, be carried through with the aid of Theorem 3 (cf. Corollary, Theorem 4). Moreover, maximal null nets could in ranking problems be formally treated as representations of incommensurability. 


\section{Application in an Information System Model}

We examine, lastly, the structure of a recursive model of a large-scale information system which was first proposed and studied in connection with a particular logical formulation of processes of communication or diffusion of information [8]. The model was originally stated in the formalism of the calculus of relations, but some of its basic properties were investigated, with greater convenience, by graph-theoretic means and associated relation matrix methods. In the earlier study, principal interest attached to the structure of transient information flow, and to algorithms for the computation of "minimum transmission times" in the propagation of information. At this point, we fix attention on issues of equilibrium or steady-state information flow. This leads naturally to an abstract connectivity approach to issues relating to the accessibility of information. In the following, we adhere for the most part to the order of development and terminology of the earlier study; some alterations of notation and language have, however, been made in the interests of clarity and conciseness $[8,10]$.

We consider a recursive system $\Omega(k)$ defined for all nonnegative integers $k$, as a composition of three fundamental binary relations, of which two are homogeneous and one nonhomogeneous. Two arbitrary and distinct sets $\Delta, \Sigma$ are assumed to be given, each finite and non-empty. A homogeneous binary relation $\rho$ called the communication relation is defined on the elements of $\Delta$. A homogeneous binary relation $\sigma$ called the derivation relation (and also called the primitive implication relation) is defined on the elements of $\Sigma ; \sigma$ is not postulated to be either reflexive or transitive. Finally, a nonhomogeneous binary relation $\tau$ called the assignment relation is defined on the product set $\Delta \times \Sigma$, with domain and range respectively contained in $\Delta$ and $\Sigma$.

An element of the field (i.e., the domain or the range) of $\rho$ is called a communication element or entity, and $x \rho y$ is read " $x$ communicates with $y . "$ An element of the field of $\sigma$ is called an information element or entity, where such entities may be of arbitrary logical or mathematical character; $\alpha \sigma \beta$ is read " $\beta$ is derived from $\alpha$ " or " $\alpha$ primitively implies $\beta$." The relation $\tau$ is regarded as an "initial input relation" in the recursive system; for $x \in \Delta, \alpha \epsilon \Sigma, x \tau \alpha$ is read " $x$ is assigned and holds $\alpha$."

The model structure is based on the perspicuous and well-known principle of computation in certain finite and closed information systems to the effect that information is produced only by the operations of (initial) assignment, communication, and derivation. In the present structure, communication and derivation are treated as equivalent or substitutable operations in respect to transmission of information and, in fact, proceed at the same unit rate. The recursive system of interest is completely defined by

$$
\Omega(0)=\tau,
$$

$$
\Omega(n)=\breve{\rho} \Omega(n-1) \cup \Omega(n-1) \sigma, \quad(n=1,2, \ldots),
$$

where $\breve{\rho}$ is the converse of $\rho . \quad \Omega(n)$ is thus given as the union of the right and left relative products of $\Omega(n-1)$ by $\sigma$ and $\breve{\rho}$ respectively, and with domain and range corresponding to that of $\tau$. The relation $\Omega(k)$ is called the thesaurus relation of order $k(k \geqslant 0)$; for any integer $k, x \in \Delta, \alpha \epsilon \Sigma, x \Omega(k) \alpha$ is read " $x$ holds $\alpha$ at stage $k$." Thus, (lb) may be regarded as providing a system analogue of modus ponens ${ }^{2}$ in the form: If $x \Omega(k) \alpha$ and $\alpha$ $\sigma \beta$, then $x \Omega(k+1) \beta \quad(x \epsilon \Delta ; \alpha, \beta \epsilon \Sigma)$. In homologous fashion, the basic triadic relation " $x$ communicates $\alpha$ to $y$ " is rendered in the form: If $x \Omega(k) \alpha$ and $x \rho y$, then $y \Omega(k+1) \alpha(x, y \in \Delta ; \alpha \epsilon \Sigma)$. The relations $\rho$ and $\sigma$ may then be regarded as expedient means of treating the fundamental operations of transfer and logical detachment. In a recent study, the relation $\tau$ of the present model has been construed as a state-description (in relation matrix form) of an information store. ${ }^{3}$ The recursive system, in effect, affords a composite representation of two processes for the propagation of information which abstracts virtually every aspect except that of connectivity.

We turn to the problem of convergence in the system defined by (la) and (lb). For this purpose, a sequence $\{\Omega(k) ; k \leqslant 0\}$ will be said to be convergent if, and only if, there exists a term $\Omega(p)$ in the sequence such that $\Omega(p)$ $=\Omega(p+1)$. Let $\Omega_{*}$ denote the union $\cup_{k=0}^{\infty} \Omega(k)$ of all terms in a sequence (convergent or not), and let $\Omega^{*}$ denote the unique limit term of a convergent sequence. $\Omega_{*}$ and $\Omega^{*}$ are respectively called the thesaurus relation closure and the thesaurus relation limit.

From (la) and (lb) we obtain in the powers of $\breve{\rho}$ and $\sigma$

$$
\Omega(m)=\bigcup_{h=0}^{m} \breve{\rho}^{h} \tau \sigma^{m-h}(m=0,1,2, \ldots),
$$

where $\breve{\rho}^{0}, \sigma^{0}$ are identity relations respectively defined on $\Delta$ and $\Sigma$. The system (1a), (1b) exhibits redundancy for $m \geqslant 2$ since

$$
\begin{aligned}
\Omega(m) & =\breve{\rho} \Omega(m-1) \cup \tau \sigma^{m} \\
& =\breve{\rho}^{m} \tau \cup \Omega(m-1) \sigma,
\end{aligned}
$$

and $(\breve{\rho} \Omega(m-1)) \cap(\Omega(m-1) \sigma) \geqslant \bigcup_{h=1}^{m-1} \breve{\rho}^{h} \tau \sigma^{m-h}$.

If both $\sigma$ and $\rho$ were at once reflexive and transitive, the thesaurus relation sequence $\{\Omega(k) ; k \geqslant 0\}$ would converge rapidly. For then by $(2), \Omega^{*}=\Omega(k)=\breve{\rho} \tau \cup$ $\tau \sigma \cup \breve{\rho} \tau \sigma$ for all $k \geqslant 2$. Moreover, $\Omega_{*}=\tau \cup \Omega^{*}$.

The general problem of convergence may be treated with advantage by the device of embedding the original system of recursions in a larger recursive system of

\footnotetext{
${ }^{2}$ Cf. Paul Hertz "Über Axiomensysteme für beliebige Satzsysteme," Math. Ann. 87 (1922), pp. 246-269.

${ }^{3}$ D. B. Hertz et al., "Research Study of Criteria and Procedures for Evaluating Scientific Information Retrieval Systems," Final Report (Contract NSF C-218), March 1962, prepared for Office of Scientific Information Service, National Science Foundation.
} 
known structure. We proceed to reformulate the original recursive system as a stationary Boolean process (discrete parameter). Let $R, S, T$ be Boolean relation matrices corresponding to $\rho, \sigma, \tau$ and let the sequence of rectangular Boolean matrices $\{W(k)$; $k \geqslant 0\}$ (called the sequence of information configurations) correspond to the sequence $\{\Omega(k) ; k \geqslant 0\}$. Moreover, let $W^{*}, W_{*}$ respectively correspond to $\Omega^{*}$ and $\Omega$. The original system is then restated as

$$
\begin{aligned}
& W(0)=T, \\
& W(n)=\breve{R} W(n-1) \cup W(n-1) S, \quad(n=1,2, \ldots),
\end{aligned}
$$

and the explicit form of $W(m)$ is given by

$$
W(m)=\bigcup_{h=0}^{m} \breve{R}^{h} T S^{m-h} \quad(m=0,1,2, \ldots) .
$$

The last system of recursions can be concisely reformulated. Let $Q$ denote the square Boolean relation matrix $\left[\begin{array}{cc}\breve{R} & T \\ \varnothing & S\end{array}\right]$, where $\varnothing$ denotes a rectangular null matrix. The new recursion constitutes a stationary Boolean process in the powers of $Q$ and yields

$$
Q^{n}=Q^{n-1} Q=\left[\begin{array}{cc}
\breve{R}^{n} & W(n-1) \\
\varnothing & S^{n}
\end{array}\right]_{(n=1,2, \ldots),}
$$

where in nonredundant manner $W(n)=\breve{R} W(n-1)$ $\cup T S^{n}=\breve{R}^{n} T \cup W(n-1) S$. The convergent behavior of the sequence $\{W(k) ; k \geqslant 0\}$ is then simply subsumed in the asymptotic behavior of the powers of $Q$. By Lemma $2 \mathrm{c}$ it is clear that a $W(k)$-sequence may converge even though $R$ or $S$ or both are oscillatory in their powers. For example, let the graph $G(Q)$ be specified as follows: (i) $G(\widetilde{R}), G(S)$ are respectively cycles of order $r$ and $s$ such that $(r, s)=1$; (ii) $G(T)$ contains only one edge. The $W(k)$-sequence then converges, and $W(r s-1)$ in $Q^{r s}$ consists exclusively of nonnull entries.

More generally, Lemma 2c provides an essentially complete graph-theoretic characterization of the convergence conditions for $W(k)$-sequences and more, in the present embedding. In fact, for $x \in \Delta, \eta \in \Sigma, x \Omega^{*} \eta$ if, and only if, for some positive integer $q_{0}$ there exist one or more cyclic traverses from vertex $\alpha_{x}$ to vertex $\alpha_{\eta}$ in $G(Q)$ (involving communication relation or derivation relation cyclic traverses) which exhibit paths of all integral lengths $q \geqslant q_{0}$. It is worth noting that a single maximal null net of $G(\vec{R})$ may contribute to two or more maximal null nets in $G(Q)$ depending on the structure of the "coupling subgraph" $G(T)$. From (3) we readily conclude by Lemma 1 and Theorem 1 that a $W(k)$-sequence converges if $R$ and $S$ are both convergent. Consequently, if $\rho, \sigma$ are both drawn from one of the following classes of relations the $W(k)$-sequence and the $\Omega(k)$-sequence exhibit convergent behavior (cf. [9]): reflexive, transitive, compact, equivalence, weak ordering, quasi-ordering, serial (strong ordering), hierarchical. From the preceding remark it also follows that the transitive closure $Q_{*}$ of $Q$ contains the union $W_{*}$, whether the $W(k)$-sequence is convergent or not.

We turn to a derivation of the explicit forms of $W^{*}$ and $W *$ in the general case. Let $\breve{R}, S$ be of dimensions $d_{r}, d_{s}$. Let $\Gamma(\breve{R}), \Gamma(S)$ denote the groups of limit matrices of $\breve{R}$ and $S$ and of periods $p_{r}, p_{s}$. Let $\breve{R}^{* *}, S^{* *}$ denote the unions of the elements of $\Gamma(\mathscr{R})$ and $\Gamma(S)$. Let $\Gamma(Q)$ denote the group of limit matrices of $Q$ of period $p_{q}$, where $p_{q} \leqslant$ l.c.m. $\left(p_{r}, p_{s}\right)$. An element $Q_{\alpha}^{\prime}$ of

$\Gamma(Q)$ will be written in the form

$$
Q^{\prime}{ }_{\alpha}=\left[\begin{array}{c}
\check{R}_{\alpha}^{\prime} W_{\alpha} \\
\varnothing S_{\alpha}^{\prime}
\end{array}\right] \quad\left(\alpha=1, \ldots, p_{q}\right),
$$

where $\breve{R_{\alpha}^{\prime}} \epsilon \Gamma(\breve{R}), S_{\alpha}^{\prime} \epsilon \Gamma(S)$. Let $Q^{* *}$ denote the union $\rho^{p} q Q_{\alpha}^{\prime}$ and let $Q_{1}^{\prime}$ denote the identity of $\Gamma(Q) ; \breve{R}_{1}^{\prime}, S_{1}^{\prime}$ $\alpha=1$

are then the identities of $\Gamma(\breve{R}), \Gamma(S)$.

The group identity $Q_{1}^{\prime}$ is idempotent so that

$$
W_{1}=\breve{R}_{1}^{\prime} W_{1} \cup W_{1} S_{1}^{\prime} \text {. }
$$

Assume now that the $W(k)$-sequence converges. The idempotent matrix $Q^{* *}$ then leads to the relation

$$
W^{*}=\breve{R}^{* *} W^{*} \cup W^{*} S^{* *} .
$$

From the equations $Q Q^{* *}=Q^{* *} Q=Q^{* *}$ we obtain

$$
\begin{aligned}
& W^{*}=\breve{R} W^{*} \cup T S^{* *}, \\
& W^{*}=W^{*} S \cup \breve{R}{ }^{* *} T .
\end{aligned}
$$

The relations $\breve{R}^{k} T S^{* *} \leqslant W^{*}, \quad \breve{R}^{* *} T S^{k} \leqslant W^{*}$ for all $k \geqslant 0$ follow from (6a) and (6b) respectively. The inclusion $\breve{R}^{* *} T S \cup \breve{R}_{*} T S^{* *} \leqslant W^{*}$ then follows readily $\left(R_{*}, S_{*}\right.$ are the transitive closures) and the converse inclusion follows directly from $\left(2^{\prime}\right)$ and (5) so that

$$
W^{*}=\breve{R}^{* *} T S_{*} \cup \breve{R}_{*} T S^{* *} .
$$

If both $\breve{R}$ and $S$ are convergent, we write $\breve{R}^{*}$ for $\breve{R}^{* *}$ $=\breve{R}_{1}^{\prime}$ and $S^{*}$ for $S^{* *}=S_{1}^{\prime}$ so that (7) becomes

$$
W^{*}=\breve{R}^{*} T S_{*} \cup \breve{R}_{*} T S^{*} .
$$

The expression for $W_{*}$ follows directly from (8) and is given by

$$
W_{*}=\breve{R}_{*} T S_{*} .
$$

From (4) it is clear that the $W(k)$-sequence converges if, and only if, $W_{1}=\breve{R}_{1}^{\prime} W_{1} \cup W_{1} S_{1}^{\prime}=\check{R}^{* *} T S_{*} \cup R_{*} T S^{* *}$. This condition may be seen to imply the following two conditions for the group unions $\breve{R}^{* *}, S^{* *}$ :

$$
\begin{aligned}
\breve{R}^{* *} T & \leqslant \breve{R}^{\prime}{ }_{1} T \cup \breve{R^{* *}} T \hat{S}, \\
T S^{* *} & \leqslant T S^{\prime}{ }_{1} \cup \hat{R} T S^{* *},
\end{aligned}
$$


the condition $W_{\alpha}=W^{*}$ can be shown to be equivalent to each of the following:

$$
\begin{aligned}
& W_{\alpha}=\breve{R}_{\alpha}^{\prime} T S . \cup \breve{R}_{*} T S^{* *}, \\
& W_{\alpha}=\breve{R}_{\Varangle} T S_{\alpha}^{\prime} \cup \breve{R}^{* *} T S_{*}, \quad\left(\alpha=1, \ldots, p_{q}\right) .
\end{aligned}
$$

Finally, for convergent $W(k)$-sequences it may be verified that $\left[\max \left(d_{s}, d_{r}\right)-1\right]^{2}+\min \left(d_{s}, d_{r}\right)$ provides a sharp upper bound for the smallest integer $q$ such that $W(q)=W^{*}$ (cf. [9]).

The expressions for $W^{*}$ and $W_{*}$ in (8) and (9) are of particular interest in the light of the present results on idempotence. The graphs $G\left(W^{*}\right)$ and $G\left(W_{*}\right)$ are by the embedding represented as subgraphs respectively of the permanent graphs $G\left(Q^{* *}\right)$ and $G\left(Q_{*}\right)$ of the composite information process. Theorems 1 and 2 (and Lemmas 1 and $2 c$ as well) consequently provide the basis for a detailed description of the thesaurus relation limit $\Omega^{*}$ and the thesaurus relation closure $\Omega_{*}$. The graph $G\left(Q^{* *}\right)$ could satisfy the requirements of Theorem 3. The formulas for $W^{*}$ and $W$ algebraically exhibit the components of information accessibility in the form of closure expressions of subsystem connectivity in $\widetilde{R}_{*}$ and $S_{*}$. By virtue of the presence of $\widetilde{R}^{* *}, S^{* *}$ in the expression for $W^{*}$ it is quite evident that the maximal nets of $G(\widetilde{R})$ and $G(S)$ constitute fundamental units of analysis in the study of the recursive system. This in turn leads to the clear possibility of constructing equivalence classes of communication elements relative to specified maximal nets of the graph of the derivation relation which may be called "information-accessibility equivalence classes." Such constructions could be effected for $W_{*}$ as well as $W^{*}$.

The matrix $W^{*}$ of a convergent sequence (for given $R, T$, and $S$ ) is viewed as the equilibrium or steadystate information configuration of the recursive system. For many significant information sequences, $W^{*}$ is identically null since $\breve{R}$ and $S$ are both null convergent, e.g., $R, S$ both strict hierarchies with graphs $G(R)$, $G(S)$ given as trees. In such cases, the closure $W_{*}$ regarded as the summary computational history of the sequence provides a useful description of the information process. In systems of greater complexity $W^{*}$ is not identically null (or the limit does not indeed exist) so that the persistence of nonnull information configurations is always mediated through "reverberating circuits" in the form of maximal cyclic nets of $G(R)$ or of $G(S)$.

The organization of information in the present recursive system may be outlined with the aid of (8) and (9), and a supplementary classification of maximal nets. Theorem 4 and the earlier noted "informationaccessibility equivalence classes" for $W^{*}$ and $W_{*}$ are also relevant here. We consider a "hierarchical" classification of the maximal nets of $G(R)$ and $G(S)$. A maximal net (null or cyclic) of $G(R)(G(S))$ is said to be of the first level if no vertex of the net is attainable from any other maximal net of $G(R)(G(S))$. A maximal net of $G(R)(G(S))$ is recursively said to be of level $k$ if, and only if, it is attainable in one step from a distinct maximal net of level $k-1$ and is not attainable in one step from any maximal net of lower level. In the general case, $G(R)$ and $G(S)$ are each possibly composed of disjoint and frequently similar (i.e., one-one corresponding) subgraphs. The transmission and distribution of information in the present recursive system for specified $R$ and $S$ are then governed by the mode of assignment of the maximal net "hierarchies" of $G(R)$ to the maximal net "hierarchies" of $G(S)$. It would, consequently, be of interest to consider alternative general system designs for the achievement of preassigned $W^{*}$ or $W_{*}$.

This work was supported in part by the U.S. Patent Office.

\section{References}

(1) F. E. Hohn and L. R. Schissler, Boolean matrices and the design of combinational relay switching circuits, Bell Syst. Tech. J. 34 177-202, (1955).

(2) M. G. Kendall, Further contributions to the theory of paired comparisons, Biometrics 11 43-62, (1955).

(3) A. G. Lunts, The application of Boolean matrix algebra to the analysis and synthesis of relay contact networks, Dokl. Akad. Nauk SSSR 70 421-423, (1950). (In Russian).

(4) O. Ore, Theory of Graphs, Am. Math. Soc., Providence (1962).

(5) C. S. Peirce, Description of a notation for the logic of relatives, resulting from an amplification of the conceptions of Boole's calculus of logic, Memoirs of the American Academy 9 317378, (1870) reprinted in Collected Papers III, 27-98 (Exact Logic) (Harvard Univ. Press, Cambridge, 1933).

(6) C. S. Peirce, Nomenclature and divisions of dyadic relations, (1903), reprinted in Collected Papers III, 366-387 (Exact Logic) (Harvard Univ. Press, Cambridge, 1933).

(7) R. T. Prosser, Applications of Boolean matrices to the analysis of flow diagrams, Tech. Rept. No. 217, Massachusetts Institute of Technology, Lincoln Laboratory, Lexington, (1960).

(8) D. Rosenblatt, A note on communication in organizations, Carnegie Institute of Technology, Pittsburgh, (unpublished) 1951.

(9) D. Rosenblatt, On the graphs and asymptotic forms of finite Boolean relation matrices and stochastic matrices, Naval Research Logistics Quarterly 4, 151-167, (1957).

(10) D. Rosenblatt, On some aspects of models of complex behavioral systems, in Information and Decision Processes, pp. 62-86 (R. E. Machol, ed.), (McGraw-Hill Book Co., New York, N.Y., 1960).

(Paper 67B4-107) 


\section{Publications of the National Bureau of Standards*}

\section{Selected Abstracts}

The maximum number of zeros in the powers of an undecomposable matrix, M. Marcus and F. May, Duke Math. J. 20, No. 4, 581-588 (Dec. 1962).

Let $A$ be an $N$-square matrix. $A$ is called decomposable if there is a permutation matrix $P$ such that $P A P^{T}$ is a subdirect sum. The question here is: given an $n$-square indecomposable matrix with complex entries, how many fixed positions $(i, j), 1 \leqslant i, j \leqslant n$ can be zero in every positive integral power of $A$. This is answered for several classes of matrices, including normal matrices with distinct characteristic roots.

Packing inequalities for circles, P. J. Davis, Michigan Math. J. 10, 25-31 (1963).

The theory of complex variables is employed to obtain inequalities for non overlapping circles.

Lower bounds to eigenvalues using operator decompositions of the form B*B, N. W. Bazley and D. W. Fox, Arch. Rat. Mech. Anal. 10, 352 (1962).

A procedure is given for obtaining lower bounds to the eigenvalues of a self-adjoint operator; the difference between the quadratic form of the operator and that of a second operator having a smaller quadratic form is assumed to be of the form $(B u, B u)^{\prime}$ in which $B$ is an operator from one Hilbert space $\mathcal{H}$ to another $\mathcal{K}^{\prime}$. The eigenvalues and eigenvectors of the smaller quadratic form are also assumed known. The lower bounds are obtained by instructing operators that have quadratic forms intermediate between the smaller and that of the given operator. The construction depends on the use of the operator $B^{*}$ adjoint to $B$.

Statistical interpretations, W. J. Youden, Book, Standard Methods of Chemical Analysis IIA, Chapt. 16, 319-325 (D. Van Nostrand Co., Princeton, New Jersey, N.J. 1963).

This paper has been prepared for the chapter on Statistics in the new (6th) edition of Scott's Standard Methods of Chemical Analysis published by Van Nostrand. The first part of the paper goes into detail regarding the steps required to obtain meaningful estimates of the errors in analytical determinations. The remainder takes up a few recurring problems in analytical chemistry along with the appropriate statistical techniques.

Precision of simultaneous measurement procedures, W. A. Thompson, Jr., J. Am. Stat. Assoc. 58, No. 302, 474-479 (June 1963). We consider the problem of measurement under the following conditions: The process of gathering the data is such that on any given item only one opportunity for measurement occurs, but it can be observed simultaneously by several instruments. The items to be measured are variable so that one cannot obtain replicate observations with the same instrument which would show directly the variance of the instrument readings. Procedures are discussed for estimating the precisions of the instruments and the variability of the items being measured.

Determining fastest routes using fixed schedules, B. M. Levin and S. Hedetniemi, Proc. Conf. American Federation for Information Processing Soc. 23, l-9 (1963).

In determining routings between locations having no direct connections, most published timetables leave to the patron the job of selecting the most desirable routing. A program has been written which determines routings using speed and cost as criteria. The solution stems from and is related to the Shortest Route Problem. This paper discusses the program and its relationship to the Shortest Route Problem.

The present program is designed for use in routing airmail. The techniques can be used to route passengers, or articles on any type of scheduled transportation trip. It can also be useful in evaluating proposed transportation networks.

Dielectric relaxation in a high temperature dipole lattice, R. Zwanzig, J. Chem. Phys. 38, No. 11, 2766 (1963).

The effect of rotational Brownian motion on the dielectric susceptibility of a rigid cubic lattice of permanent point dipoles is calculated. All dipolar interactions are taken into account by means of a high temperature perturbation expansion. The most significant result is that dipolar interactions give rise to additional new relaxation times, increasing dielectric loss at high frequencies.

Some free products of cyclic groups, M. Newman, Michigan Math. J. 9, 369-373 (1962).

Let $\lambda_{n}=2 \cos (\pi / n)$. The principal result of this paper is that the group generated by the linear fractional transformations

$$
\tau^{\prime}=-1 /\left(\tau-\lambda_{p}\right), \tau^{\prime}=-1 /\left(\tau+\lambda_{q}\right)
$$

is the free product of a cyclic group of order $p$ and a cyclic group of order $q$ for $p \geqslant 2, q \geqslant 3$.

Multipliers of difference sets, M. Newman, Can. J. Math. 121-124 (Nov. 1961).

Let $D$ be a difference set with parameters $v, k, \lambda, v>k>\lambda>0$. Let $q$ be a prime divisor of $n=k-\lambda$. The Hall-Ryser theorem states that if $(q, v)=1$ and $q>\lambda$ then $q$ is a multiplier of $D$. In this paper a proof of theorem is given by incidence matrices alone. The restriction $q>\lambda$ is also removed in certain cases, e.g. $n=q$, or $n=2 q$ and $(v, 7)=1$.

The segemental variation of Blaschke products, G. T. Cargo, Duke Math. J. 30, No. 1, 143-150 (Mar. 1963).

We say that a function which is regular in the open unit disk $D$ has finite segmental variation at a point $e^{i \theta}$ provided every line segment connecting $e^{i \theta}$ to a point of $D$ is mapped onto a rectifiable curve by the function.

Seidel and Walsh proved that a univalent function has finite segmental variation almost everywhere; and Tsuji proved that, if a function has a finite Dirichlet integral, it has finite segmental variation off a set whose (logarithmic) capacity is zero.

In this paper, analogous local and global theorems are established for a well-known class of functions whose members are not univalent and do not have finite Dirichlet integrals, namely, (infinite) Blaschke products.

The structure of some subgroups of the modular group, M. Newman, Illinois J. Math. 6, No. 3, 480-487 (Sept. 1962).

The groups $\Gamma^{m}$ generated by the $m$ th powers of all elements of the $2 \times 2$ modular group $\Gamma$ are studied and their structure determined. The connection with the Burnside problem is discussed.

Conditions for second-order waves in hypo-elasticity, B. Bernstein, Trans. Soc. Rheology VI, No. 1, 263-273 (1962).

The study in hypo-elasticity of second order waves, regarded as propagating singular surfaces of order two in displacement, yields results similar to those in general elasticity theory. The amplitude of a wave must be an eigenvector of a certain matrix, the acoustical tensor, which depends on the stress and direction of propagation, and the product square of the wave speed with the mass density must be the corresponding eigenvalue. Conditions are stated that at least one wave is possible in each direction and that the existence of a longitudinal amplitude imply the possibility of a longitudinal wave. The condition that the acoustical tensor be always symmetric is the same as that a Cauchy-elastic hypo-elastic material possess a strain energy.

The invariance of symmetric functions of singular values, M. Marcus and H. Minc, Pacific J. Math. 12, No. 1, 327-332 (1962). 
Let $M$ denote the vector space of all $m \times n$ matrices over the complex numbers and let $T$ be a linear map of $M$ into itself. Let $f(A)$ denote the $r^{\text {th }}$ elementary symmetric function of the squares of the singular values of $A, A \in M$. We determine completely the structure of the group $M_{f}$ of all linear $T$ satisfying the invariance condition: $f(T(A))=$ $f(A)$ for all $A \epsilon M$.

Bounds for cofactors and arithmetic minima of quadratic forms, M. Newman, J. London Math. Soc. 38, p. 215-217 (Apr. 1963). Let $A$ be a real, symmetric $n \times n$ positive definite matrix of determinant $d$. It is shown that after a suitable integral congruence transformation bounds for the determinants of the leading principal minor matrices of $A$ and for the diagonal elements of $A$ may be derived which depend only on $n$ and $d$. It is also shown that the Hermite constant $\gamma_{n}$ satisfies

$$
\gamma_{m+n}^{m+n} \geqslant \gamma_{m}^{m} \gamma_{n}^{n}
$$

Convergence to normality of powers of a normal random variable, N. Severo and L. J. Montzingo, Jr., Bull. Intern. Stat. Inst. XXXIX, Pt. 2, 491-500 (1962).

Let $Y$ be a normally distributed random variable, and let $p$ be a real number such that $y^{p}$ is also real, $-\infty \leqslant y \leqslant+\infty$. Let $\mu_{y}$ and $\sigma_{y}$ be the mean and standard deviation of $Y$, respectively. Let $X=Y^{p}$. It is shown that if $p$ is a positive integer, then $X$ is asymptotically normally distributed with mean $\mu_{x}$ and standard deviation $\sigma_{x^{\prime}}$ as $\mu_{y} / \sigma_{y} \rightarrow \infty$. Otherwise, $X$ is asymptotically normally distributed with mean $\mu_{0}=\mu_{y}^{p}\left[1+0\left(\eta^{-2}\right)\right]$ and standard deviation

$$
\sigma_{0}=\left(|p| \mu_{y}^{p / \eta}\right)\left[1+0\left(\eta^{-2}\right)\right]^{1 / 2}
$$

as $\mu_{y} / \sigma_{y} \rightarrow \infty$. When $\mu_{x}$ and $\sigma_{x}$ exist, it is shown that $\mu_{y} / \sigma_{y} \rightarrow \infty$ is necessary and sufficient to insure that $\mu_{x} / \sigma_{x} \rightarrow \infty$.

Non-linear effects in spectra of the iron group, R. E. Trees, Phys. Rev. 129, No. 3, 1220-1224. (Feb. 1, 1963).

As known for the last ten years, calculations made with the linear theory agree poorly with experiment in the $3 d^{6}$ configuration of $\mathrm{Fe}$ III, compared to the agreement obtained for the $3 d^{5} 4 s$ configuration of that spectrum. It is shown that effects of $3 s 3 d^{6} 4 s$ on $3 s^{2} 3 d^{5} 4 s$ satisfy the necessary conditions for linear behavior, consistent with the close agreement obtained in $3 d^{5} 4 s$ of Fe III. Effects of $3 s 3 d^{7}$ on $3 s^{2} 3 d^{6}$ are not linear; it is shown that if they are accounted for in the calculations, then close agreement is obtained in the $3 d^{6}$ configuration also (the mean error is $\pm 65 \mathrm{~cm}^{-1}$ ). The magnitude of the parameter $\gamma$, introduced to define this non-linear interaction, is found to be $40 \%$ smaller than expected from Hartree-Fock calculations, but this is a minor inconsistency in view of the known inadequacies of s.c.f. orbitals. It is pointed out that the parameters of the linear theory are changed considerably when the non-linear effects are accounted for.

Normal functions, the Montel property, and interpolation in $\mathbf{H}^{\infty}, \mathrm{G}$. T. Cargo, Michigan Math. J. 10, 141-146 (1963).

This paper exhibits an interrelationship among three apparently unrelated subjects, namely, normal functions, Montel's property, and interpolation with bounded holomorphic functions in the open unit disk. As a by-product, new and somewhat shorter proofs of several known results are obtained.

Copositive and completely positive quadratic forms, $\mathrm{M}$. Hall, Jr., and M. Newman, Proc. Camb. Phil. Soc. 59, 329-339 (1963). The relation between quadratic forms which are sums of squares of non-negative linear forms, and quadratic forms which are non-negative for non-negative values of the variables is investigated and a duality theorem is proved.

Covers and packings in a family of sets, J. Edmonds, Bull. Am. Math. Soc. LXVIII, No. 5, 494-499 (Sept. 1962).

A graph-theoretic characterization is given of (1) minimum-cardinality covers of a set $S$ chosen from a specified family $F$ of its subsets and (2) maximum-cardinality families of mutually disjoint sets chosen from $F$.

On the pedestrian queueing problem, G. Weiss, Bull. Intern. Stat. Inst. XXXIX, No. 4, 163-168 (1962).

J. C. Tanner has discussed the problem of the delay to pedestrians trying to cross a road, and being blocked by traffic. In his treatment it was assumed that the pedestrians arrived at the intersection completely at random and crossed either upon arrival or in a group, after the departure of a car. Tanner also assumed that the criterion for crossing depended on whether the gap seen by the pedestrian exceeded a given one, $T_{c}$, and that the gaps were described by a Poisson distribution. Mayne generalized this problem by allowing arbitrary gap and arrival distributions with the gap acceptance criterion given by a critical gap $T_{c}$, as in Tanner's treatment. We shall consider the same problem assuming a Poisson arrival distribution for pedestrians, but allowing for a probabilistic gap acceptance criterion, i.e., we let $\alpha(t)$ be the probability that a pedestrian will cross the road if he sees a gap of $t$ in the oncoming traffic. In this paper the imbedded Markoff process generated by this situation will be studied, an expression for the generating function for the number of pedestrians at the intersection will be given, and various other statistics discussed.

A connection between Tauberian theorems and normal functions, G. T. Cargo, Bull. Am. Math. Soc. 68, No. 4, 400-401 (July 1962).

In this note it is pointed out that certain Tauberian theorems follow immediately from some recent research of Lehto-Virtanen and Bagemihl-Seidel.

Note on a subgroup of the modular group, M. Newman and J. R. Smart, Proc. Am. Math. Soc. 14, No. 1, 102-104 (Feb. 1963).

Let $\Gamma$ be the $2 \times 2$ modular group, $\Gamma^{2}$ the subgroup of $\Gamma$ generated by the squares of the elements of $\Gamma, \Gamma^{3}$ the subgroup of $\Gamma$ generated by the cubes of the elements of $\Gamma$. Let $G^{\prime}$ denote the commutator subgroup of $G$ for any subgroup $G$ of $\Gamma$. Then it is shown that

$$
\left(\Gamma^{2}\right)^{\prime}\left(\Gamma^{3}\right)^{\prime}=\Gamma^{\prime},
$$$$
\left(\Gamma^{2}\right)^{\prime} \cap\left(\Gamma^{3}\right)^{\prime}=\Gamma(6)
$$

where $\Gamma(6)$ is the principal congruence subgroup of $\Gamma$ of level 6 .

Linear velocity-gradient term in time-dependent pair distribution function, R. E. Nettleton, J. Chem. Phys. 38, No. 8,1791802 (Apr. 1963).

A Chapman-Enskog type perturbation solution, in gradients of energy, velocity, and density, is proposed for the hierarchy of integro-differential equations obeyed by the time-dependent UrsellMayer functions. The hierarchy is then terminated by an ansatz relating the three-particle Ursell functions to those of lower order, which yields a closed system for the perturbations to the singlet and pair distribution functions (it is assumed the equilibrium functions are known). Attention is focused on the equation for the twoparticle functions, and on the term therein proportional to the divergence of fluid velocity, from which the bulk viscosity may be calculated. An expansion for this term is assumed in powers of scalar products of particle separations and velocities, in which the coefficients in turn are expanded in Sonine polynomials in the velocities. The functions which multiply these polynomials satisfy a system of integral equations, for which a first approximation is written down and the solution discussed. Finally, it is shown that substitution of the solution for the pair distribution function into the hierarchy equation for the singlet distribution yields an infinite number of conditions which may be used to evaluate a corresponding number of parameters in the ansatz employed to close the hierarchy.

\section{Other NBS Publications}

J. Res. NBS 67A (Phys. and Chem.), No. 5 (Sept.-Oct. 1963) 70 cents.

Reduction of space groups to subgroups by homogeneous strain. H. S. Peiser, J. B. W achtman, Jr., and R. W. Dickson.

High-temperature thermodynamic functions for zirconium and unsaturated zirconium hydrides. T. B. Douglas.

Heat of oxidation of aqueous sulfur dioxide with gaseous chlorine W. H. Johnson and J. R. Ambrose.

Thickness of adsorbed polystyrene layers by ellipsometry. R. R. Stromberg, E. Passaglia, and D. J. Tutas.

Melting temperature and change of lamellar thickness with time for bulk polyethylene. J. J. Weeks.

Precise coulometric titrations of potassium dichromate. G. Marinenko and J. K. Taylor. 
Resolution of limits of analyzers and oscillatory systems. E. L. R. Corliss.

Synthesis, purification, and physical properties of seven twelvecarbon hydrocarbons. T. W. Mears, C. L. Stanley, E. L. Compere, Jr., and F. L. Howard.

Reactions of polyfluorobenzenes with nucleophilic reagents. L. A. Wall, W. J. Pummer, J. E. Fearn, and J. M. Antonucci.

J. Res. NBS 67C (Eng. and Instr.), No. 4 (Oct.-Dec. 1963) 75 cents.

Limitations on electron beam density of unipotential electron guns at low voltages. J. A. Simpson and C. E. Kuyatt.

New radiofrequency mass spectrometer having high duty cycle. R. H. Mills.

A power-series buildup factor formulation. Application to rectangular and off-axis disk source problems. J. B. Hubbell.

An alinement interferometer. J. B. Saunders.

Determination of optical path difference for a photographic objective. F. E. Washer and W. R. Darling.

Development of filters for a thermoelectric colorimeter. S. H. Emara and R. P. Teele.

Application of air bearings to an electrodynamic vibration standard. T. Dimoff and B. F. Payne.

Leak-resistant rotation seal for vacuum applications. F. L. Howard. Studies on the tungsten-rhenium thermocouple to $2000{ }^{\circ} \mathrm{C}$. D. B. Thomas.

J. Res. NBS 67D (Radio Prop.), No. 5 (Sept.-Oct. 1963) 70 cents.

Ionospheric VHF scattering near the magnetic equator during the International Geophysical Year. R. Cohen and K. L. Bowles.

Radio pulse propagation by a reflection process at the lower ionosphere. J.R. Johler.

Field of a horizontal magnetic dipole in the presence of a magnetoplasma halfspace. G. Tyras, A. Ishimaru, and H. M. Swarm.

Reflection of VLF radio waves from an inhomogeneous ionosphere. Part II. Perturbed exponential model. J. R. Wait and L. C. Walters.

Collisional detachment and the formation of an ionospheric $C$ region. E. T. Pierce.

Magnetic torques and Coriolis effects on a magnetically suspended rotating sphere. J. C. Keith.

Radiation field characteristics of lightning discharges in the band $1 \mathrm{kc} / \mathrm{s}$ to $100 \mathrm{kc} / \mathrm{s}$. W. L. Taylor

Low-frequency radio propagation into a moderately rough sea. D. F. Winter.

VLF superdirective loop arrays. E. W. Seeley.

Curves of ground proximity loss for dipole antennas (a digest). L. E. Vogler and J. L. Noble.

Observations and results from the "hiss recorder" an instrument to continuously observe the VLF emissions. J. M. Watts, J. A. Koch, and R. M. Gallet.

J. Res. NBS 67D (Radio Prop.), No. 6 (Nov.-Dec. 1963) 70 cents. A radiometeorological study, part I. Existing radio-meteorological parameters. J. A. Lane and B. R. Bean.

A radiometeorological study, part II. An analysis of VHF field strength variations and refractive index profiles. B. R. Bean, V. R. Frank, and J. A. Lane.

A radiometeorological study, part III. A new turbulence parameter. B. R. Bean, E. J. Dutton, J. A. Lane, and W. B. Sweezy.

Reflection of radio waves from undulating tropospheric layers. A. T. Waterman, Jr., and J. W. Strohbehn.

Oblique propagation of groundwaves across a coastline-Part I. J. R. Wait.

Oblique propagation of groundwaves across a coastline-Part II. J. R. Wait and C. M. Jackson.

On the index of refraction of air, the absorption and dispersion of centimeter waves by gases. G. Boudouris. Translated from French by G. W. Curtis.

A numerical approach to the solution of radio diffraction problems. J. K. Hargreaves and S. Hargreaves.

Inversion of radio wave absorption data to establish ionospheric properties. I. Nondeviative absorption. A. D. Wheelon.

Radiation in a lossless magneto-ionic medium at frequencies high relative to the electron gyrofrequency. J. W. Marini.

Radiation through cylindrical plasma sheaths. J. H. Harris.

Chart for determining the effects of ionospheric tilts using an idealized model. T. A. Croft and R. B. Fenwick.
Reflection of VLF radio waves from an inhomogeneous ionosphere. Part III. Exponential model with hyperbolic transition. J. R. Wait and L. C. Walters.

Families of distributions for hourly median power and instantaneous power of received radio signals. M. M. Siddiqui and G. H. Weiss.

Statistical methods in radar astronomy. Determination of surface roughness. H. S. Hayre.

Electrical conductivity of the Great Lakes. L. H. Doherty.

Standard X-ray diffraction powder patterns, H. E. Swanson, M. C. Morris, R. P. Stinchfield, and E. H. Evans, NBS Mono. 25, Section 2 (May 3, 1963) 35 cents.

Tensile and impact properties of selected materials from 20 to $300{ }^{\circ} \mathrm{K}$, K. A. Warren and R. P. Reed, NBS Mono. 63 (June 28, 1963) 35 cents.

Refractive indices and densities of aqueous solutions of invert sugar, C. F. Snyder and A. T. Hattenburg, NBS Mono. 64 (June 7, 1963 15 cents.

Reduction of data for piston gage pressure measurements, J. L. Cross, NBS Mono. 65 (June 17, 1963) 15 cents.

Tabulation of data on receiving tubes, C. P. Marsden and J. K. Moffitt, NBS Handb. 83 (May 23, 1963) \$1.25. (Supersedes Handb. 68.)

Radiobiological dosimetry. Recommendations of the International Commission on radiological units and measurements, NBS Handb. 88 (April 30, 1963), Supersedes parts of Handb. 78. Handbooks 84 through 89 will completely replace $\mathrm{H} 78,25$ cents.

Quarterly radio noise data, September, October, November 1962, W. Q. Crichlow, R. T. Disney, and M. A. Jenkins, NBS Tech. Note 18-16 (June 10, 1963) 60 cents.

Mean electron density variations of the quiet ionosphere, November 1959, J. W. Wright, L. R. Wescott, and D. J. Brown, NBS Tech. Note 40-9 (April 22, 1963) 35 cents.

Mean electron density variations of the quiet ionosphere, December 1959, J. W. Wright, L. R. Wescott, and D. J. Brown, NBS Tech. Note 40-10 (March 24, 1963) 35 cents.

Phototypesetting of computer output, an example using tabular data, W. R. Bozman, NBS Tech. Note 170 (June 25, 1963) 10 cents.

Curves of ground proximity loss for dipole antennas, L. E. Vogler and J. L. Noble, NBS Tech. Note 175 (May 20, 1963) 30 cents.

Table of attenuation error as a function of vane-angle error for rotary vane attenuators, W. Larson, NBS Tech. Note 177 (May 20, 1963) 75 cents.

An interpolation procedure for calculating atmospheric band absorptions from laboratory data, L. Droppleman, L. R. Megill, and R. F. Calfee, NBS Tech. Note 178 (June 3, 1963) 20 cents.

Tabulation of published data on Soviet electron devices, C. P. Marsden, NBS Tech. Note 186 (June 3, 1963) 45 cents.

Impedance of commercial Leclanché dry cells and batteries, R. J. Brodd and H. J. Dewane, NBS Tech. Note 190 (July 5, 1963) 40 cents.

Tables describing small-sample properties of the mean, median, standard deviation, and other statistics in sampling from various distributions, C. Eisenhart, L. S. Deming, and C. S. Martin, NBS Tech. Note 191 (June 14, 1963) 20 cents.

Calculations of the potential and effective diffusion constant in a polyelectrolyte solution, S. R. Coriell and J. L. Jackson, NBS Tech. Note 192 (June 28, 1963) 25 cents.

A bibliography of foreign developments in machine translation and information processing, J. L. Walkowicz, NBS Tech. Note 193 (July 10, 1963) \$1.00.

National standard reference data program, background information, NBS Tech. Note 194 (June 1963) 25 cents.

\section{Publications in Outside Journals}

Nuclear resonance and the hyperfine field in dilute alloys of nickel in iron, R. L. Streever, L. H. Bennett, R. C. La Force, and G. F. Day, J. Appl. Phys. 34, No. 4, Pt. 2, 1050-1051 (Apr. 1963).

The history of Pt. 27, E. Wichers, Book, Temperature, Its Measurement and Control in Science and Industry 3, Pt. 1, 259-262 (Reinhold Publ. Corp., New York, N.Y., 1962).

Some causes of resonant frequency shifts in atomic beam machines. I. Shifts due to other frequencies of excitation, J. H. Shirley, 
J. Appl. Phys. 34, 783-788 (Apr. 1963).

Some causes of resonant frequency shifts in atomic beam machines. II. The effect of slow frequency modulation on the Ramsey line shape, J. H. Shirley, J. Appl. Phys. 34, 789-791 (Apr. 1963).

On the dependence of absorption coefficients upon the area of the absorbent material, E. D. Daniel, J. Acoust. Soc. Am. 35, No. 4, 571-573 (Apr. 1963).

The role of the International Union of pure and applied chemistry, E. Wichers, J. Chem. Doc. 3, No. 7, 7-11 (1963).

New scale of nuclidic masses and atomic weights, E. Wichers, Nature 194, No. 4829, 621-624 (May 19, 1962).

Note on a subgroup of the modular group, M. Newman and J. R. Smart, Proc. Am. Math. Soc. 14, No. 1, 102-104 (Feb. 1963).

Calibration of photogrammetric lenses and cameras at the National Bureau of Standards, F. E. Washer, Photogrammetric Eng. XXIX, No. 1, 113-119 (Jan. 1963).

The orthobaric densities of parahydrogen, derived heats of vaporization and critical constants, H. M. Roder, D. E. Diller, L. A. Weber, and R. D. Goodwin, Cryogenics 3, 16-22 (Mar. 1963).

Melting pressure equation for the hydrogens, R. D. Goodwin, Cryogenics 2, No. 6, 1-3 (Dec. 1962).

Isotopic fractionation of uranium in sandstone, J. N. Rosholt, W. R. Shields, and E. L. Garner, Science 139, 224-226 (Jan. 18, 1963).

The total electron content of the ionosphere at middle latitudes near the peak of the solar cycle, R. S. Lawrence, D. J. Posakony, O. K. Garriott, and S. C. Hall, J. Geophys. Res. 68, 1889-1898 (Apr. 1, 1963).

Present status of our knowledge of atomic transition probabilities, W. L. Wiese, Proc. Tenth Colloquium Spectroscopic Intern., pp. 37-56 (Univ. of Maryland, College Park, Md., 1962).

Rubber and rubber products, W. P. Tyler and M. Tryon, Book, Industrial and Natural Products and Noninstrumental Methods, 6th Ed., Standard Methods of Chemical Analysis IIB, Chapt. 43, 2146-2226 (D. Van Nostrand Co., Inc., New York, N.Y., 1963).

The formation and oxidation of high-area carbon films, V. R. Deitz and E. F. McFarlane, Proc. Fifth Carbon Conference II, 219-232 (Pergamon Press, Inc., London, England, 1963).

Pure substance and measurement, E. Wichers, Mater. Res. Stds. 1, No. 4, 314-315 (Apr. 1961).

The specific heat at constant volume of parahydrogen at temperatures from 15 to $90^{\circ} \mathrm{K}$ and pressures to $340 \mathrm{~atm}, \mathrm{~B}$. A. Younglove and D. E. Diller, Cryogenics 2, No. 6, 1-5 (Dec. 1962).

Radiation detectors, L. Costrell, Science 139, No. 3558, 899 (Mar. 8, 1963).

Electron spin resonance of gamma-irradiated cellulose, R. E. Florin and L. A. Wall, J. Polymer Sci. 1, Pt. A, 1163-1173 (1963).

The speed of light, A. G. McNish, IRE Trans. Instr. I-11, Nos. 3 \& 4, 138-148 (Dec. 1962).

Fundamentals of measurement, A. G. McNish, Electro-Technol. 53, 113-128 (May 1963).

Optimum estimators of the parameters of negative exponential distributions from one or two order statistics, M. M. Siddiqui, Ann. Math. Stat. 34, 117-121 (Mar. 1963).

Building a simple transistor tester, G. F. Montgomery, Electronics 36, No. 16, 56 (Apr. 19, 1963).

Electron microscopy studies of the surfaces of magnetic recording media, F. Nesh and D. B. Ballard, IEEE Trans. Audio AU-11, No. 1, 15-18 (Jan. 2, 1963).

A magnetic amplifier for use with diode logic, E. W. Hogue, Proc. IEEE 1963 Intern. Conf. Nonlinear Magnetics No. T-149, 8.6-1 to 8.6-6 (Apr. 1963).

Kinetics of the acid-catalyzed hydrolysis of acetal in water-acetone solvents at 15,25 , and $35^{\circ}$, R. K. Wolford, J. Phys. Chem. 67, 632-636 (1963).

Performance characteristics of split-type residential air-to-air heat pumps, J. C. Davis and P. R. Achenbach, Suppl. Bull. Inst. Intern. Refrigeration, pp. 1-7 (1961-1962).

Crystallographic changes with the substitution of aluminum for iron in dicalcium ferrite, D. K. Smith, Acta Cryst. 15, 1146-1152 (Jan. 1963).

Methods for the analysis of rubber and related products, M. Tryon and E. Horowitz, Handb. Analytical Chemistry, Sect. 13, pp. 233256 (McGraw-Hill Book Co., Inc., New York, N.Y., 1963).

Pressure-density-temperature relations of freezing liquid para hydrogen to 350 atmospheres, R. D. Goodwin, Cryogenics 3, 12-15 (Mar. 1963).

Nuclear magnetic resonance in metal powders at low tempera- tures, R. J. Snodgrass and L. H. Bennett, J. Appl. Spectry. 17, No. 2, 53-54 (1963).

Millimeter wavelength resonant structures, R. W. Zimmerer, M. V. Anderson, G. L. Strine, and Y. Beers, IEEE Trans. Microwave Theory Tech. MTT-11, 141-149 (Mar. 1963).

Lunar point-to-point communication, L. E. Vogler, Book, Technology of Lunar Exploration, Progress in Astronautics and Aeronautics 9, 533-559 (Academic Press Inc., New York, N.Y., 1963).

Comment on "Parametric behavior of an ideal two-frequency varactor", G. F. Montgomery, Proc. IEEE 51, No. 3, 491 (Mar. 1963).

Distribution of latitude of red arcs, E. Marovich and F. E. Roach, J. Geophys. Res. 68, No. 7, 1885-1888 (Apr. 1, 1963).

Instability of the equatorial $F$ layer after sunset, W. Calvert, J. Geophys. Res. 68, 2591-2593 (May 1, 1963).

A simple environmental chamber for rotating beam fatigue testing machines, J. A. Bennett, Mater. Res. Std. 3, No. 6, 480-482 (June 1963).

Standard potential of the silver-silver chloride electrode and activity coefficients of hydrochloric acid in aqueous methanol $(33.4 \mathrm{Wt}$. $\%$ ) with and without added sodium chloride at $25^{\circ}, \mathrm{R}$. G. Bates and D. Rosenthal, J. Phys. Chem. 67, 1088-1090 (1963).

International standardization. A new responsibility of the engineer, A. T. McPherson, Natl. Acad. Sci.-Natl. Res. Council Div. of Eng. and Ind. Res. Newsletter, No. 26, 2-4 (June 1, 1963).

Path antenna gain and comments on "properties of $400 \mathrm{Mc} / \mathrm{s}$ longdistance tropospheric circuits", W. J. Hartman, Proc. IEEE 51, 847-848 (May 1963).

Absorption and scattering of photons by deformed nuclei, E. G. Fuller, Proc. Second All-Union Conf. Nuclear Reactions at Low and Intermediate Energies, p. 419, 1960 (Russian Academy of Science, U.S.S.R., 1962).

Microwave spectrum of tertiary butyl chloride, A. Comparison of tertiary butyl structures, D. R. Lide, Jr., and M. Jen, J. Chem. Phys. 38, No. 7, 1504-1507 (Apr. 1, 1963).

Search for a slow component in alpha ionization, Z. Bay and R. M. Pearlstein, Phys. Rev. 130, No. 1, 223-227 (Apr. 1963).

Evidence regarding the mechanism of fatigue from studies of environmental effects, J. A. Bennett, Acta Met. 11, No. 7, 799800 (July 1963).

Dielectric friction on a rotating dipole, R. Zwanzig, J. Chem. Phys. 38, No. 7, 1605-1606 (Apr. 1, 1963).

Field-aligned $E$-region irregularities identified with acoustic plasma waves, K. L. Bowles, B. B. Balsley, and R. Cohen, J. Geophys. Res. 68, 2485-2501 (May 1, 1963).

The association of plane-wave electron-density irregularities with the equatorial electrojet, R. Cohen and K. L. Bowles, J. Geophys. Res. 68, 2603-2611 (May 1, 1963).

Calorimetric calibration of the electrical energy measurement in an exploding wire experiment, D. H. Tsai and J. H. Park, Exploding Wires 2, 27-107 (Plenum Press, Inc., New York, N.Y., 1962).

Quasi-equilibrium theory of mass spectra, H. M. Rosenstock and M. Krauss, Book, Mass Spectrometry of Organic Ions, pp. 1-64 (Academic Press, Inc., New York, N.Y., 1963).

Determination of differential X-ray photon flux and total beam energy, J. S. Pruitt and H. W. Koch, Book, Nuclear Physics 5, Chapt. 2.8.2, Pt. B, 508-553, Ed. L. C. L. Yuan and C. Wu (Academic Press, Inc., New York, N.Y., 1963).

Index to the communications of the ACM, 1958-1962, W. W. Youden, Comm. ACM 6, No. 3, 1-32 (Mar. 1963).

Electrode potentials in fused systems. VI. Membrane potentials, K. H. Stern, Phys. Chem. 67, 893-895 (1963).

Electron spin resonance spectra of aged, $\gamma$-irradiated polystyrenes, R. E. Florin, L. A. Wall, and D. W. Brown, J. Polymer Sci. Pt. A. 1, 1521-1529 (1963).

Magnetic properties of acetinide element alloys and compounds, J. C. Eisenstein, Book, Landolt-Bornstein, 6Ed. 2, Pt. 9, p. 3.236 (Springer-Velag, Berlin, 1962).

Planar twin boundary in aluminum, T. H. Orem, Trans Met. Soc. AIME 227, 786-788 (June 1963).

Radio noise anomalies in August 1958, C. A. Samson, J. Geophys. Res. 68, 2719-2726 (May 1, 1963).

Optical studies of the formation and breakdown of passive films formed on iron single crystal surfaces in inorganic inhibitor solutions, J. Kruger, J. Electrochem. Soc. 110, No. 6, 654-663 (June 1963).

The importance of environment in fatigue failure of metals, J. A. Bennett, W. L. Holshouser and H. P. Utech, Book, Fatigue of 
Aircraft Structures, pp. 1-18 (Pergamon Press, Inc., London, England, 1963).

Production of Lyman alpha radiation in ion-atom collisions, G. H. Dunn, R. Geballe, and D. Pretzer, Phys. Rev. 128, No. 5, 22002206 (ec. 1, 1962).

Microwave spectrum of aluminum monofluoride, D. R. Lide, Jr., J. Chem. Phys. 38, No. 8, 2027 (Apr. 15, 1963).

Some limits on the effect of coronal self-emission upon the excitation state of coronal ions, R. N. Thomas and C. Pecker, Astrophys. J. 137, No. 3, 967-980 (1962).

Oil baths for saturated standard cells, P. H. Lowrie, Jr., ISA J. 9, No. 12, 47-50 (Dec. 1962).

Two-stream plasma instability as a source of irregularities in the ionosphere, D. T. Farley, Jr., Phys. Rev. Letters 10, No. 7, 279282 (Apr. 1, 1963).

Performance of the barium fluoride film hygrometer element on radiosonde flights, F. E. Jones, J. Geophys. Res. 68, No. 9, 27352751 (May 1, 1963).

Radiation induced polymerization of propylene at high pressure, D. W. Brown and L. A. Wall, J. Phys. Chem. 67, 1016-1019 (1963).

Dielectric friction on a moving ion, R. Zwanzig, J. Chem. Phys. 38, No. 7, 1603-1605 (Apr. 1, 1963).

Survey of $\mathrm{Rb}^{85} / \mathrm{Rb}^{87}$ ratios in minerals, W. R. Shields, E. L. Garner, C. E. Hedge and S. S. Goldich, J. Geophys. Res. 68, No. 8, 23312334 (Apr. 15, 1963).

Dissociation constant of pyrrolidinium ion and related thermodynamic quantities from 0 to $50^{\circ}, \mathrm{H}$. B. Hetzer, R. G. Bates, and R. A. Robinson, J. Phys. Chem. 67, 1124-1127 (1963).

Introduction to the International Symposium on Equatorial Aeronomy, R. Cohen and K. L. Bowles, J. Geophys. Res. 68, 2359-2361 (May 1, 1963).

Interference fringes with long path difference using He-Ne laser, T. Morokuma, K. F. Nefflen, T. R. Lawrence, and T. M. Klucher, J. Opt. Soc. Am. 53, No. 3, 394-395 (Mar. 1963).

Hydrogen retention system for pressure calibration of microphones in small couplers, W. Koidan, J. Acoust. Soc. Am. 35, No. 4, 614 (Apr. 1963).

Numerical computation of time-dependent properties of isotopically disordered one-dimensional harmonic crystal lattices, R. J. Rubin, J. Phys. Soc. Japan 18, Suppl. II, 63-69 (1963).

The National Bureau of Standards: Where measurement is the central theme, C. S. McCamy, Ind. Phot. 11, No. 8, 28 (Aug. 1962). Statistical computation of configuration and free volume of a polymer molecule with solvent interaction, J. Mazur and L. Joseph, J. Chem. Phys. 38, No. 6, 1292-1300 (Mar. 15, 1963).

A comparison of two independent atomic time scales, J. Newman, L. Fay, and W. R. Atkinson, Proc. IEEE 51, No. 3, 498-499 (March 1963).

Symmetry conditions for internal friction caused by jumping of point defects in crystals, J. B. Wachtman, Jr., and H. S. Peiser, J. Appl. Phys. Letter 1, No. 1, 20-22 (Sept. 1962).

Change in occlusion of complete dentures caused by a pipe habit: a case report, J. B. Woefel and G. C. Paffenbarger, J. Am. Dental Assoc. 66, No. 4, 478-485 (Apr. 1963).

Measurement of a maximum in the isothermal crystallization ratetemperature curve for polypentene-1, F. A. Quinn, Jr., and J. Powers, Polymer Letters (J. Polymer Sci., Pt. B) 1, No. 7, 341-344 (July 1963).

Pressure induced shifts of infrared lines due to polar molecules, E. K. Plyler, M. A. Hirshfield, and J. H. Jaffe, J. Chem. Phys. 38, No. 1, 257-258 (Jan. 1963).

Radio spectrum of SH, H. E. Radford and M. Linzer, Phys. Rev. Letters 10, No. 10, 443-444 (May 15, 1963).

Electrophoretic deposits of barium titanate, V. A. Lamb and H. I. Salmon, J. Am. Ceram. Bull. 41, No. 11, 781 (1962).

Measurement of the lattice constants of neon isotopes in the temperature range $4^{\circ}-24^{\circ} \mathrm{K}, \mathrm{L}$. H. Bolz and F. A. Mauer, Proc. 1lth Annual Conf. Applications of X-ray Analysis, Denver Res. Inst. (Plenum Press, Inc., New York, N.Y., 1962).

Thermodynamic properties of gases, J. Hilsenrath, Am. Inst. Phys. Handb. 2d Ed., Sec. 4h, 101-157 (1963).

The decoration of dislocations in aluminum oxide, H. E. Bond and K. B. Harvey, J. Appl. Phys. 34, No. 2, 440-44l (Feb; 1963).

The effect of geomagnetic crochet on cosmic ray intensity, C. S. Warwick, J. Geophys. Res. 68, No. 10, 3303-3304 (May 15, 1963).

Design of an interferometric oil manometer for vacuum measurement, A. M. Thomas, D. P. Johnson, and J. W. Little, 1962 Trans. Ninth Natl. Vacuum Symp., Am. Vacuum Soc. pp. 468--473 (1962).
Transference numbers in pure molten sodium nitrate, R. J. Labrie and V. A. Lamb, J. Electrochem. Soc. 110, No. 7, 810-814 (July 1963).

Unstable detonation near a hypervelocity missile, F. W. Ruegg and W. W. Dorsey, Ninth Symp. Intern. Combustion, pp. 476-477 (1963).

Vacuum-tight cylinder joints and ball-and-socket joints, L. A. Guildner and H. F. Stimson, Rev. Sci. Instr. 34, No. 6, 658-659 (June 1963).

Exchange processes in the reaction of boron trichloride with triethylamine-boron trifluoride, T. D. Coyle, Proc. Chem. Soc. (London), p. 172 (June 1963).

Analysis of the third spectrum of praseodymium, J. Sugar, J. Opt. Soc. Am. 53, No. 7, 831-838 (July 1963).

Study of Knudsen's method of pressure division as a means of calibrating vacuum gauges, S. Schuhmann, Trans. Ninth Natl. Vacuum Symp. Am. Vacuum Soc., pp. 463-467 (1962).

Design of low voltage electron guns, J. A. Simpson and C. E. Duyatt, Rev. Sci. Instr. 34, No. 3, 265-268 (Mar. 1963).

Classification of two-electron excitation levels of helium, J. W. Cooper, U. Fano, and F. Prats, Phys. Rev. Letters 10, No. 12, 518-521 (June 1963).

Photodisintegration of light nuclei, E. Hayward, Rev. Mod. Phys. 35, No. 2, 324-331 (Apr. 1963).

Solid state physics, H. P. R. Frederikse (Editor and Part Author), Book, Am. Inst. Phys. Handbook Sec. 9, 2d Ed. (McGraw-Hill Book Co., New York, N.Y., 1963).

Very low temperature data. Properties of paramagnetic salts, R. P. Hudson, AIP Handb. 2d Ed. Sec. 5g, pp. 222-223 (1963).

VLF propagation - theory and experiment, J. R. Wait, Monograph, Electromagnetic Waves in Stratified Media, Chapt. IX, pp. 264288 (Pergamon Press, Inc., Oxford, England, 1962).

Plastics, G. M. Kline, Americana annual, p. 535 (Americana Corp., Chicago, Ill., 1963).

Sum rules relating coherent X-ray scattering data to the diamagnetic nuclear shielding constant and to the self-energy of the charge distribution of the scatterer, J. N. Silverman and Y. Obata, J. Chem. Phys. 38, No. 5, 1254-1255 (1963).

Present status of our knowledge of atomic transition probabilities, W. L. Wiese, Proc. Xth Colloq. Spectroscopicum Intern., pp. 37-56 (Ed. E. R. Lippincott and M. Margoshes, Spartan Books, Washington, D.C., 1963).

Collective correlation of plasma, C. M. TchenCzechoslovak J. Phys., Proc. 2d Conf. Electronics XXI, No. 5-6, 516-521 (Apr. 1962).

Double-photon photodetachment of negative ions, S. Geltman, Phys. Rev. Letters 4, No. 3, 168-169 (Apr. 1, 1963).

Mathematical analysis of thermal environment in underground shelters, P. R. Achenbach, T. Kusuda, and F. J. J. Drapeau, ASHRAE Symp. Survival Shelters, pp. 9-33 (June 1962).

Effect of insulation on the weathering of smooth-surfaced, built-up roofs exposed to solar heating, W. C. Cullen and W. H. Appleton, Am. Roofer and Building Improvement Building Contractor Mag. Pt. I, 53, No. 3, 19-22 (Mar. 1963); Pt. II, 53, No. 4, 14 (Apr. 1963).

The precise evaluation of lens distortion, F. E. Washer, Photogrammetric Eng. XXXI 327-332 (Mar. 1963).

Pressure broadening as a prototype of relaxation, U. Fano, Phys. Rev. 131, No. 1, 259-268 (July 1, 1963).

The atomic spectra of the rare earths: their presence in the sun, C. E. Moore, Appl. Opt. 2, No. 7, 665-674 (July 1963).

The conductive-disk method of measuring the thermal conductivity of insulators, H. E. Robinson, Suppl. Bull. Intern. Inst. Refrigeration, Annexe 1962-1, 43-50 (Aug. 1962).

Electrodeposition of alloys. Principles and practice. Vol. I. General Survey, principles, and alloys of copper and of silver, and Vol. II, Practical and specific information, A. Brenner, Book, Academic Press, Inc., New York, N.Y., 1963.

Factorial designs and the direct product, B. Kurkjian and M. Zelen, Bull. Intern. Stat. Inst. XXXIX, Pt. 2, 509-519 (1962).

Electron affinity of atomic iodine, B. Steiner, M. L. Seman and L. M. Branscomb, J. Chem. Phys. 37, No. 6, 1200-1202 (June 1962).

The contact properties of thin films on semiconductors, T. Higier and G. G. Harman, November 1961 Contact Symp. Proc. Univ. Maine (Mar. 20, 1963).

Temperature scales, thermocouples and resistance thermometers, H. F. Stimson, J. F. Swindells, and R. E. Wilson, Am. Inst. Phys. Handb. 2d Ed., Sec. 4a, pp. 2-21 (1963).

Field-emission study of carbon monoxide on tantalum, R. Klein and L. B. Leder, J. Chem. Phys. 38, No. 8, 1866-1872 (Apr. 15, 1963). Field emission observations of carbon on tantalum, R. Klein and L. 
Leder, J. Chem. Phys. 38, No. 8, 1863-1866 (Apr. 1963).

Electron impact ionization of atomic hydrogen, S. Geltman, M. R. Rudge, and M. J. Seaton, Proc. Phys. Soc. 81, Pt. 2, No. 520, 375-377 (Feb. 1, 1963).

Electron energy levels and their relationship to lattice defects in reduced rutile, J. H. Becker and W. R. Hosler, (Proc. Intern. Conf. Crystal Lattice Defects), J. Phys. Soc. Japan 18, Suppl. II, 152160 (1963).

Addendum: Vibration-rotation spectra of BrCN, A. Maki, J. Chem. Phys. 38, No. 5, 1261-1262 (May 1963).

The present status of radiometric calibrations in the ultraviolet spectrum, R. Stair, Symp. on Military Applications of Ultraviolet Radiation, Rept. No. LAS-TR-199-37 (Nov. 1962).

Acoustic properties of liquids, M. Greenspan, Am. Inst. Phys. Handb. 2d Ed. Sec. 3e, pp. 71-82 (1963).

Reflection mechanisms for sporadic $E$, K. Tao and J. R. Wait, Book, Ionospheric Sporadic E, Ed. E. K. Smith and S. Matsushita, Chapt. 4, Pt. II, pp. 13-27 (Pergamon Press, Inc., Oxford, England, 1962).

Gas measurement and other services of the National Bureau of Standards, C. T. Collett, Am. Gas Assoc. Preprint DMC-63, pp. 1-3 (1963).

Review of reviews of atomic spectra, W. Meggers, Appl. Opt. 2, No. 7, 657-663 (July 1963).

Molecular parameters of ethane, W. J. Lafferty and E. K. Plyler, J. Chem Phys. 37, No. 11, 2688-2692 (Nov. 1962).

New autioionizing atomic energy levels in $\mathrm{He}, \mathrm{Ne}$, and $\mathrm{A}, \mathrm{R} . \mathrm{P}$. Madden and K. Codling, Phys. Rev. Letters 10, No. 12, 516-518 (June 15, 1963)

A modification of the Born-Mayer potential function as applied to the crystalline alkali halides, T. B. Douglas, J. Chem. Phys. 38, No. 10, 2461-2466 (May 15, 1963).

Discussion of errors of a recommended standard resistor-noise test system, N. Newman and G. T. Conrad, Jr., IRE Trans. Professional Group on Component Parts CP-9, No. 4, 180-192 (Dec. 1962).

Microbalance techniques for high temperature applications - further developments, N. J. Carrera and R. F. Walker, Book, Vacuum Microbalance Techniques, 3, 153-177 (Plenum Press, Inc., New York, N.Y., 1963).

Research in American Society for Testing Materials, B. L. Wilson, Mater. Res. Std. 2, No. 8, 694-695 (Aug. 1962).

Heat capacities, G. T. Furukawa and T. B. Douglas, Am. Inst. Phys. Handb. 2d Ed., Sec. 4e, pp. 47-63 (1963).

High resolution spectra in the region from 2 to $6 \mu$, E. K. Plyler and E. D. Tidwell, (Proc. Intern. Molecular Conf.), Book, Advances in Molecular Spectroscopy, pp. 1336-1342 (Pergamon Press Inc., New York, N.Y., 1962).

Impressions of Japan, R. S. Marvin, Japanese J. Kobunshi (High Polymers), Soc. High Polymers 12, No. 130, 53 (1963).

Theory of the electronic polarizabilities of ions in crystals: Application to the alkali halide crystals, A. R. Ruffa, Phys. Rev. 130, No. 4, 1412-1423 (May 15, 1963).

Annual report for IAU commission 14, C. E. Moore, Trans. Astronomical Union XIA97-XIB, 208 (1962).

Atomic flame reactions involving $\mathrm{N}$-atoms, $\mathrm{H}$-atoms and ozone, $\mathrm{D}$ Garvin and H. P. Broida, 9th Intern. Combustion Symp., pp. 678-688 (1963)

The effect of moisture on heat transfer through insulated flat-roof constructions, F. J. Powell, Suppl. Bull. Intern. Inst. Refrigeration, Annexe 1962-1, 61-70 (Aug. 1962).

Infrared spectrum of acetylene- $d_{1}$, W. J. Lafferty, E. K. Plyler, and E. D. Tidwell, J. Chem. Phys. 37, No. 9, 1981-1988 (Nov. 1962).

Nickel and alloys, M. R. Meyerson, Encyclopedia of Engineering Materials and Processes, pp. 439-442 (Reinhold Publ. Corp., New York, N.Y., 1963)

Audiofrequency dispersion effects in lanthanide salts at low temperatures, R. P. Hudson and B. W. Mangum, Book, Magnetic and Electric and Electric Resonance and Relaxation, Ed. J. Smidt, pp. 135-146 (North-Holland Publ. Co., Amsterdam, The Neitherlands, 1963).
Some problems in temperature measurements from line spectra, J. T. Jefferies, Book, Temperature, Its Measurement and Control in Science and Industry 3, No. 1, 703-711 (1962).

Nonreciprocity of propagation of VLF radio waves along the magnetic equator, D. D. Crombie, Proc. IEEE 51, No. 4, 617-618 (Apr. 1963).

Infrared spectrum of carbon tetrafluoride, A. Maki, E. K. Plyler, and R. Thibault, J. Chem. Phys. 37, No. 8, 1899-1900 (Oct. 1962).

Matrix effects in the gaseous $\mathrm{H}$ atom - condensed olefin system; surface reaction-olefin diffusion model, R. Klein and M. D. Scheer, J. Phys. Chem. 66, No. 12, 2677-2681 (Dec. 1962).

A Note on domain conversions of $\mathrm{BaTiO}_{3}, \mathrm{P}$. H. Fang and W. S. Brower, J. Appl. Phys. 34, No. 5, 1516 (May 1963).

Stability relations of dravite, a tourmaline, C. R. Robbins and H. S. Yoder, Jr., Geophysical Laboratory Yearbook 1961-62 (Note) (Carnegie Institute of Washington, Feb. 1963).

An alignment interferometer, J. B. Saunders, Appl. Opt. 2, No. 5, 541 (May 1963).

An application of the peek-a-boo principle to information retrieval, J. Stern, Proc. Symp. Materials Information Retrieval, pp. 93-115 (Nov. 28-29, 1962).

Critical review of sugar color and turbidity measurements, F. G. Carpenter and V. R. Deitz, Sugar Ind. Tech. 21, Paper 65, 32-52 (1962).

Molecular vib-rotors. The theory and interpretation of high resolution infrared spectra, H. C. Allen, Jr., and P. C. Cross, Book, John Wiley \& Sons, Inc., New York, N.Y., 1963.

On the vacuum ultraviolet refectance of evaporated aluminum before and during oxidation, R. P. Madden, L. R. Canfield, and G. Haas, J. Opt. Soc. Amm. 53, No. 5, 620-625 (May 1963).

An LCAO-MO-SCF study of the structure of $\mathrm{HO}_{2}$, M. E. Boyd, Program Comm. Intern. Symp. on Molecular Structure and Spectroscopy, pp. B404-1-B404-4 (Science Council of Japan, Tokyo, 1962).

Burning behavior of building finish materials: Two-test methods, A. R. Robertson, Spec. Publ. Fed. Fire Council, pp. 21-35 (Apr. 1962).

The kinetics of description II: $\mathrm{Ca}^{+}$and $\mathrm{Ba}^{+}$from rhenium, M. D. Scheer and J. Fine, J. Chem. Phys. 38, No. 2, 307 (Jan. 1963).

A computer for the Abel inversion, C. R. Yokley and J. B. Shumaker, Rev. Sci. Instr. 34, No. 5, 551-557 (May 1963).

Rotation-vibration constants of acetylene, E. K. Plyler, E. D. Tidwell, and T. A. Wiggins, J. Opt. Soc. Am. 53, No. 5, 589-593 (May 1963).

Calculation of crystallite size distributions from X-ray line broadening, A. Bienenstock, J. Appl. Phys. 34, No. 5, 1391 (May 1963).

Osmotic and activity coefficients of tetraethylammonium iodide in aqueous solution at $25^{\circ}$, V. E. Bower and R. A. Robinson, Trans. Faraday Soc. 59, No. 488, 1717-1719 (Aug. 1963).

Dependence of the electrical conductivity and thermoelectric power of pure and aluminum-doped rutile on equilibrium oxygen pressure and temperature, J. Yahia, Phys. Rev. 130, No. 5, 1711-1719 (June 1, 1963).

A portable impedance tube, R. D. Berendt and H. A. Schmidt, Jr., J. Acoust. Soc. Am. 35, No. 7, 1049-1052 (July 1963).

Inertial seismograph design-limitations in principle and practice, B. S. Melton and D. P. Johnson, Proc. IRE 50, No. 11, 2328-2339 (Nov. 1962).

Nuclear moment of $\mathrm{Ni}^{61}$ from nuclear resonance studies in steady external magnetic fields, R. L. Streever, Phys. Rev. 128, No. 4, 1632-1633 (Nov. 15, 1962).

Electromagnetic waves in stratified media, J. R. Wait, Book, Pergamon Press, Inc., New York, N.Y., 1962.

Absolute magnetic susceptibilities by the Gouy and the ThorpeSenftle methods, G. A. Candela and R. E. Mundy, IRE Trans. Instr. I-11, No. 3-4, 106-109 (Dec. 1962).

The following papers were published in the Proc. Intern. Conf. Ionosphere, London, 1962 (The Institute of Physics and The Physical Society, London, England):

A model of the atmosphere and the ionosphere in the $E$ and $F 1$ regions, R. B. Norton, T. E. VanZandt, and J. S. Denison, pp. $26-34$. 
Doppler studies of the ionospheric effects of solar flares, K. Davies, pp. 76-83.

Ionospheric variations during geomagnetic storms, S. Matsushita, pp. $120-127$.

The location of the irregularities responsible for ionospheric scintillation of a radio source, H. J. A. Chivers, pp. 258-266.

Equatorial spread- $F$ motions, W. Calvert, K. Davies, E. Stiltner, and J. T. Brown, pp. 316-322.

Evidence for field-aligned ionization irregularities between 400 and $1000 \mathrm{~km}$ above the earth's surface, W. Calvert, T. E. VanZandt, R. W. Knecht, and G. B. Goe, pp. 324-329.

Experimental observations and theoretical calculations leading to a model for the lower ionosphere, R. H. Doherty, pp. 428-434.
Radio wave reflections at a continuously stratified plasma with electron collision frequency proportional to energy and arbitrary magnetic induction, J. R. Johler, pp. 436-445.

Very low frequency propagation in the earth-ionosphere waveguide of non-uniform width, J. R. Wait, pp. 446-451.

*Publications for which a price is indicated are available by purchase from the Superintendent of Documents, U.S. Government Printing Office, Washington, D.C., 20402 (foreign postage, one-fourth additional). Reprints from outside journals and the NBS Journal of Research may often be obtained directly from the authors. 